To appear in the International Journal of Control

Vol. 00, No. 00, Month 20XX, 1-28

\title{
Event-triggered Control for Semi-global Stabilization of Systems with Actuator Saturation
}

\author{
Liangyin Zhang ${ }^{\mathrm{a}}$ and Michael Z. Q. Chen ${ }^{\mathrm{a} *}$ \\ ${ }^{a}$ Department of Mechanical Engineering, The University of Hong Kong, Hong Kong.
}

(Received 00 Month 20XX; accepted 00 Month 20XX)

\begin{abstract}
This paper investigates the problem of event-triggered control for semi-global stabilization of null controllable systems subject to actuator saturation. First, for a continuous-time system, novel event-triggered low-gain control algorithms based on Riccati equations are proposed to achieve semi-global stabilization. The algebraic Riccati equation with a low-gain parameter is utilized to design both the event-triggering condition and the linear controller; a minimum inter-event time based on the Riccati ordinary differential equation is set a priori to exclude the Zeno behavior. In addition, the high-low gain techniques are utilized to extend the semi-global results to event-based global stabilization. Furthermore, for a discretetime system, novel low-gain and high-low-gain control algorithms are proposed to achieve event-triggered stabilization. Numerical examples are provided to illustrate the theoretical results.
\end{abstract}

Keywords: Actuator saturation; event-triggered control; Riccati equation; semi-global stabilization.

\section{Introduction}

In practical control systems, actuators are always subject to saturation, making the issue of actuator saturation an important research problem for control science and engineering (Bernstein \& Michel, 1995). When a system contains no strictly unstable modes, it can be globally stabilized in spite of the saturation (Teel, 1992; Yang et al., 1997). Furthermore, linear control can be adopted for semiglobal stabilization (Lin et al., 1996; Teel, 1995). The global stabilization means that the domain of attraction of the closed-loop system under the control law is the entire state space. The semiglobal stabilization means that the system is stabilized by a one-parameter family of control laws whose domain of attraction can tend to the entire state space (Grognard et al., 2002). Recently, the actuator saturation issue is considered in network synchronization (Chen et al., 2015b; Su et al., 2013, 2014), where semi-global synchronization is achieved. The semi-global output regulation subject to input saturation is achieved by composite nonlinear control in (Wang et al., 2014). The control for nonlinear Markov jumping systems with input saturation is studied in (Chen et al., 2014a). And the saturation problem is studied for singular Lipschitz systems in (Zuo et al., 2015). The extensive studies on systems subject to actuator saturation demonstrate that the saturation problem is important in control theory and applications.

Event-based sampling and control, which originate from the research on aperiodic sampling (Gupta, 1963), have been extensively studied since the late 1990s (Aström \& Bernhardsson, 1999; Arzén, 1999). This has led to the gradually established event-triggered control (ETC), which can prevent unnecessary samplings as well as information transmissions and require less control updates than the traditional periodic control method. The ETC theory is first systematically studied in (Tabuada, 2007) based on the Lyapunov stability theory. An event-triggering rule is guaranteed to be legitimate in the sense that the inter-event time is lower bounded such that accumulative events

\footnotetext{
*Corresponding author. Email: mzqchen@hku.hk.
} 
known as the Zeno behavior (Ames et al., 2006) do not exist. The event-driven controllers are studied for linear systems in (Heemels et al., 2008). The self-triggered control is proposed in (Anta \& Tabuada, 2010; Wang \& Lemmon, 2009) to reduce the cost of continuous-time monitoring of the state. And the event-trigger strategy is applied to sensor/actuator networks and generalized to a decentralized form in (Mazo \& Tabuada, 2011), where a minimum time $\tau$ is set a priori instead of being guaranteed by the local event-triggering functions to ensure the legitimacy. In addition, the distributed ETC is analyzed in (Wang \& Lemmon, 2011); the event-triggered cooperative control is studied in (Chen et al., 2014b; Zhang et al., 2014b); and the ETC for discrete-time network synchronization is addressed in (Chen et al., 2015a). The discrete-time ETC is first studied in (Eqtami et al., 2010). And in (Heemels et al., 2013), the periodic ETC is proposed for linear systems to combine the advantages of both ETC and the traditional sampled control. The extensive studies on event-triggered control motivate the presented work of ETC with input saturation.

Recently, the ETC strategy is applied to systems subject to actuator saturation to achieve local stabilization. In (Lehmann \& Johansson, 2012), the event-triggered PI control is studied for a scalar system with actuator saturation. In (Kiener et al., 2014), the anti-windup compensation is investigated to enlarge the domain of attraction of systems with actuator saturation. In (Wu et al., 2014), the event-triggered control is studied for the discretized systems with actuator saturation, which is similar to the periodic ETC (Heemels et al., 2013). The controller synthesis is studied by solving the matrix optimization problem to enlarge the domain of attraction (Wu et al., 2014). In (Ni et al., 2015), both ETC and self-triggered control are studied for input-saturated systems. In (Kiener et al., 2014; Ni et al., 2015; Wu et al., 2014), the linear matrix inequalities (LMI) are utilized to design the ETC laws based on a quadratic event-triggering function. The LMIs are involved with the event-trigger parameter, making the design dependent on the selection of the parameter. In addition, to guarantee the feasibility of the ETC algorithm, the initial states of the unstable system are required to be located within the domain of attraction which is generally a bounded neighborhood of the system equilibrium point.

In this paper, the problem of event-based linear semi-global stabilization of null controllable systems subject to input saturation is investigated. The results here can be found in (Zhang, 2015). First, for a continuous-time system, novel low-gain ETC algorithms are proposed to achieve semiglobal stabilization. The algebraic Riccati equation (ARE) with a low-gain parameter is utilized to design both the event-triggering condition and the linear controller; a minimum inter-event time $\tau$ based on the Riccati ordinary differential equation is set a priori to prevent the Zeno behavior. Furthermore, for a discrete-time system, novel event-triggered control algorithms based on the discrete-time ARE are proposed to achieve semi-global stabilization. When the initial system value is known to be located within a bounded set, the low-gain parameter can be appropriately selected for both continuous-time and discrete-time systems such that unnecessary control updates are reduced while the closed-loop stability is preserved under bounded control protocol. In addition, based on the gain-scheduling methods in (Grognard et al., 2002; Shi et al., 2013), the high-low gain techniques are utilized to extend the semi-global results to event-based global stabilization.

The contribution and significance of the results in this paper are two-fold: (i) low-gain ETC algorithms with novel event-triggering conditions are designed for both continuous-time and discretetime systems such that unnecessary control updates are further reduced than the ETC law based on a simple quadratic event-triggering function; (ii) ARE is utilized to design the low-gain controllers and to construct the Lyapunov functions such that the event-trigger parameters can be freely selected while the closed-loop stability can always be guaranteed.

The remaining of the paper is organized as follows. In Section 2, the problem of event-based semi-global stabilization is formulated. The main results for continuous-time systems are presented in Sections 3. The main results for discrete-time systems are established in Section 4. The numerical examples are provided in Section 5. Finally, conclusion is drawn in Section 6.

Nomenclature: Throughout this paper, $\mathbb{R}^{p}$ and $\mathbb{R}^{p \times q}$ represent the $p$-dimensional real vector space and the set of all $p \times q$ real matrices, respectively. For $x \in \mathbb{R}^{p},\|x\|$ denotes its Euclidian norm; and $\|x\|_{\infty} \triangleq \max _{i}\left|x_{i}\right|$. For $X \in \mathbb{R}^{p \times p}$, its eigenvalues are denoted by $\lambda_{i}(X)$ satisfying that 
$\left|\lambda_{1}(X)\right| \leq \ldots \leq\left|\lambda_{p}(X)\right|$; and $\rho(X)=\left|\lambda_{p}(X)\right|$ denotes its spectral radius. For $M \in \mathbb{R}^{p \times q}, M^{\mathrm{T}}$ denotes its transpose and $\|M\| \triangleq \sqrt{\rho\left(M^{\mathrm{T}} M\right)}$ denotes its spectral norm. The $p \times p$ identity matrix is denoted by $I_{p}$. A square matrix $A$ is said to be Hurwitz if all its eigenvalues have negative real parts $\operatorname{Re}\left(\lambda_{i}(A)\right)<0 ; A$ is said to be Schur if $\rho(A)<1$. A pair $(A, B)$ is stabilizable if there exists an $F$ such that $(A-B F)$ is stable, where $A \in \mathbb{R}^{n \times n}$ and $B \in \mathbb{R}^{n \times m}$. The notation $X \succ Y$ (respectively, $X \succeq Y)$ means that $(X-Y)$ is positive definite (respectively, positive semi-definite). The saturation function with threshold of $\varpi$ is defined as $\operatorname{sat}_{\varpi}(u) \triangleq \operatorname{sgn}(u) \min \{|u|, \varpi\}$.

\section{Problem Statement}

Consider the following system subject to actuator saturation:

$$
x^{+}=A x(t)+B \cdot \pi(u(t))
$$

where $x \in \mathbb{R}^{n}, u \in \mathbb{R}^{m} ; x^{+} \triangleq \dot{x}(t)$ for continuous-time system and $x^{+} \triangleq x(t+1)$ for discretetime system; $(A, B)$ is stabilizable; and $\pi: \mathbb{R}^{m} \rightarrow \mathbb{R}^{m}$ is a saturation operator defined as $\pi(u) \triangleq$ $\left[\pi_{1}\left(u_{1}\right), \ldots, \pi_{m}\left(u_{m}\right)\right]^{\mathrm{T}}$, with the saturation function $\pi_{i}\left(u_{i}\right) \triangleq \operatorname{sat}_{\varpi}\left(u_{i}\right)=\operatorname{sgn}\left(u_{i}\right) \min \left\{\left|u_{i}\right|, \varpi\right\}$, where $\varpi>0$ is an input-saturation threshold given a priori.

The problem of event-based semi-global stabilization is as follows: for any given bounded set $\mathcal{X} \subset \mathbb{R}^{n}$, design an event-triggering condition to generate an event-triggered updating time sequence $\left\{t_{0}, t_{1}, \ldots\right\}$, and design a one-parameter family of linear feedback laws $u(t)=-K(\varepsilon) x\left(t_{k}\right)$, which use only the feedback information at the updating time $t_{k}, k=0,1, \ldots$, such that $\lim _{t \rightarrow \infty} x(t)=0$ as long as $x(0) \in \mathcal{X}$, and $\mathcal{X}$ can tend to the entire space $\mathbb{R}^{n}$ as $\varepsilon$ approaches zero. In this paper, the low-gain technique (Lin, 1999) will be utilized to design the bounded control protocol so that actuator saturation never occurs.

In the proposed event-triggered control algorithms for both continuous-time and discrete-time systems, the error variable $e(t)$ will be used. For $t \in\left[t_{k}, t_{k+1}\right), k \geq 0$, denote

$$
e(t)=x\left(t_{k}\right)-x(t)
$$

For the continuous-time system, a lower bound has to be guaranteed for the inter-event time so that the Zeno behavior is prevented. In this paper, a minimum inter-event time is set a priori in the event-trigger strategy so that the event trigger is active only after $t=t_{k}+\tau$.

It is well known that semi-global stabilization cannot be achieved for a control system containing strictly unstable open-loop dynamics subject to actuator saturation (Lin et al., 1996; Teel, 1995). Then, it is assumed that $A$ is marginally stable.

\section{Low-gain Design for Continuous-time Systems}

In this section, system (1) is treated as a continuous-time system subject to actuator saturation. A continuous-time event-trigger strategy is proposed in Section 3.1. A bounded control protocol is designed in Section 3.2. The semi-global stabilization result is established in Section 3.3. The high-low gain design for global stabilization is studied in Section 3.4.

\subsection{Event-trigger Strategy}

In this subsection, the event-triggered mechanism is described to generate the updating time sequence $\left\{t_{k}\right\}$ for continuous-time systems.

Algorithm 1: Event-based updating: 
Step 1. If $x(0) \neq 0$, set $t_{0} \triangleq 0$; otherwise, both the controller and the event trigger are inactive. At the beginning of each updating process, $t=t_{k}, k \geq 0$, the feedback control input $u$ is updated. A minimum inter-event time $\tau>0$, which will be designed in Section 3.3, is set a priori. During the time interval $\left[t_{k}, t_{k}+\tau\right)$, the event trigger is inactive.

Step 2. At the time $t=t_{k}+\tau$, the event trigger is activated. An event-triggering function $f(t)$ satisfying $f\left(t_{k}\right) \leq 0$ will be designed in Section 3.2. Either if $f\left(t_{k}+\tau\right)>0$, or if $f\left(t_{k}+\tau\right)=0$ while $\left\|x\left(t_{k}+\tau\right)\right\| \neq 0$, the next event is triggered at $t_{k+1}=t_{k}+\tau$; if $f\left(t_{k}+\tau\right)<0$, for $t>t_{k}+\tau$, the next updating event is triggered at instant $t_{k+1}>t_{k}+\tau$ when $f\left(t_{k+1}\right)=0$ and $f(t)<0$ for all $t \in\left[t_{k}+\tau, t_{k+1}\right)$; if $f\left(t_{k}+\tau\right)=\left\|x\left(t_{k}+\tau\right)\right\|=0$, stabilization has been achieved in a finite time; if $f(t)<0$ for all $t \in\left[t_{k}+\tau,+\infty\right)$, denote $t_{k+1} \triangleq+\infty$. The feedback control will be designed in the linear form of $u(t)=-K x\left(t_{k}\right), t \in\left[t_{k}, t_{k+1}\right)$.

Step 3. When a finite $t_{k+1}$ is triggered, a new updating cycle will begin, then go to Step 1.

Remark 1: This algorithm guarantees that for all possible $k \geq 0, x(t)$ is continuously differentiable on $\left(t_{k}, t_{k+1}\right)$, has the right derivative $x_{+}^{\prime}$ at $t_{k}$, and is continuous at $t_{k+1}$; when saturation does not occur, $x(t)$ is second-order differentiable on $\left(t_{k}, t_{k+1}\right)$ and has the second-order right derivative $x_{+}^{\prime \prime}$ at $t_{k}$.

\subsection{Bounded Control Protocol}

The following assumption plays an important role in the continuous-time stabilization through bounded control.

Assumption 1: The pair $(A, B)$ is asymptotically null controllable under bounded control; that is, (i) the pair $(A, B)$ is stabilizable; (ii) all the eigenvalues of $A$ are located within the closed left-half complex plane. In addition, since control is unnecessary for stabilization when $A$ is Hurwitz, it is assumed that $A$ is not Hurwitz and $B \neq 0$.

Lemma 1: (Lin, 1999) Let Assumption 1 hold, $R \succ 0$, and $Q(\varepsilon)$ be a parameter-dependent positive definite matrix satisfying the monotonic convergence $\lim _{\varepsilon \rightarrow 0^{+}} Q(\varepsilon)=0$. Then, for each $\varepsilon>0$, there exists a unique positive definite matrix $P=P(\varepsilon)$ that solves the algebraic Riccati equation (ARE)

$$
A^{\mathrm{T}} P+P A-P B R^{-1} B^{\mathrm{T}} P+Q(\varepsilon)=0 .
$$

Moreover, $\left(A-B R^{-1} B^{\mathrm{T}} P\right)$ is Hurwitz; and $\lim _{\varepsilon \rightarrow 0^{+}} P(\varepsilon)=0$.

The design of a bounded control protocol for continuous-time system (1) is performed in three steps.

Algorithm 2: Event-based low-gain stabilization:

Step 1. Find $P(\varepsilon) \succ 0$ to solve the ARE

$$
A^{\mathrm{T}} P(\varepsilon)+P(\varepsilon) A-P(\varepsilon) B B^{\mathrm{T}} P(\varepsilon)+Q(\varepsilon)=0,
$$

where $\varepsilon>0$ is a low-gain parameter to be designed and $Q(\varepsilon)$ is a parameter-dependent positive definite matrix satisfying the monotonic convergence $\lim _{\varepsilon \rightarrow 0^{+}} Q(\varepsilon)=0$. 
Step 2. Denote that

$$
K(\varepsilon) \triangleq B^{\mathrm{T}} P(\varepsilon)
$$

For brevity, $K(\varepsilon)$ is denoted as $K$ in the sequel. By Lemma 1 and Assumption 1 , one has that $(A-B K)$ is Hurwitz and $\|K\|>0$. Then, the event-triggering function is designed as

$$
\begin{aligned}
& f(t)=\max \left\{f_{1}(t), f_{2}(t)\right\}, \\
& f_{1}(t)=-\sigma x(t)^{\mathrm{T}} Q(\varepsilon) x(t)-\sigma x(t)^{\mathrm{T}} K^{\mathrm{T}} K x(t)-2 x(t)^{\mathrm{T}} K^{\mathrm{T}} K e(t), \\
& f_{2}(t)=\|K e(t)\|-\theta\|K x(t)\|,
\end{aligned}
$$

where the event-trigger parameters $\sigma, \theta \in(0,1)$ are appropriately selected.

Step 3. A feedback law using $x\left(t_{k}\right)$ is designed as

$$
u(t)=-K x\left(t_{k}\right), t \in\left[t_{k}, t_{k+1}\right), k \geq 0 .
$$

Lemma 2: The unique positive definite solution $P(\varepsilon)$ to ARE (3) and the controller matrix $K$ in (4) satisfy that $\left\|B^{\mathrm{T}} P(\varepsilon) B\right\| \cdot P(\varepsilon) \succeq K^{\mathrm{T}} K$.

Proof. It is straightforward to verify that $\left\|B^{\mathrm{T}} P(\varepsilon) B\right\|=\left\|P(\varepsilon)^{\frac{1}{2}} B B^{\mathrm{T}} P(\varepsilon)^{\frac{1}{2}}\right\|$. Then, one has

$$
\begin{aligned}
\left\|B^{\mathrm{T}} P(\varepsilon) B\right\| P(\varepsilon) & =P(\varepsilon)^{\frac{1}{2}}\left(\left\|P(\varepsilon)^{\frac{1}{2}} B B^{\mathrm{T}} P(\varepsilon)^{\frac{1}{2}}\right\| I_{n}\right) P(\varepsilon)^{\frac{1}{2}} \\
& \succeq P(\varepsilon)^{\frac{1}{2}}\left(P(\varepsilon)^{\frac{1}{2}} B B^{\mathrm{T}} P(\varepsilon)^{\frac{1}{2}}\right) P(\varepsilon)^{\frac{1}{2}}=K^{\mathrm{T}} K .
\end{aligned}
$$

\subsection{Event-based semi-global stabilization}

The main continuous-time result for event-based semi-global stabilization is presented in the following theorem.

Theorem 1: Consider continuous-time system (1) subject to actuator saturation with saturation threshold $\varpi>0$. Let Assumption 1 hold. Then, Algorithms 1 and 2 can achieve semi-global exponential stabilization of system (1). That is, for any given bounded set $\mathcal{X} \subset \mathbb{R}^{n}$, and any $\sigma, \theta \in(0,1)$ in the event-triggering function (5), there exist an $\varepsilon^{*} \in(0,1]$ for ARE (3) and a lower bound $\tau>0$ for the inter-event time, such that with the low-gain parameter $\varepsilon=\varepsilon^{*}, \lim _{t \rightarrow \infty} x(t)=0$ is achieved exponentially as long as $x(0) \in \mathcal{X}$.

Proof. The key point for the proof is to establish that if $x\left(t_{k}\right) \neq 0, k \geq 0$, then for all $t \in\left[t_{k}, t_{k}+\tau\right]$, (i) $\|x(t)\|>0$; (ii) $\|e(t)\| \leq \delta\|x(t)\|$ with $\delta$ to be defined in Step 3 of the proof; (iii) $\|K x(t)\|_{\infty} \leq \varpi$. The rationale for this procedure is: (i) utilizing the event-triggering conditions; (ii) using the fact that $e\left(t_{k}\right)=0$; (iii) setting the low-gain parameter such that $V(x(0))$ satisfies a condition to be given in Step 3 of the proof. As a result, it will be obtained that on the interval $\left[t_{k}, t_{k}+\tau\right)$, $V=x^{\mathrm{T}} P(\varepsilon) x$ is decreasing and $\|K x(t)\|_{\infty} \leq \varpi$, despite that the event trigger is inactive.

Step 1. Closed-loop Dynamics. 
By (1), (2) and (6), one has $u(t)=-K(e(t)+x(t))$ and $\dot{e}=-\dot{x}$. When saturation does not occur, the closed-loop dynamics will be

$$
\dot{x}(t)=A_{F} x(t)+B_{F} e(t), \dot{e}(t)=-A_{F} x(t)-B_{F} e(t),
$$

where $A_{F} \triangleq A-B K$ and $B_{F} \triangleq-B K$.

For the stability analysis of (1), the following quadratic Lyapunov function is used:

$$
V(x(t)) \triangleq x(t)^{\mathrm{T}} P(\varepsilon) x(t)
$$

which is continuously differentiable on $\left(t_{k}, t_{k+1}\right)$, has the right derivative $V_{+}^{\prime}$ at $t_{k}$, and is continuous at $t_{k+1}$. When saturation does not occur, by (3), (5), (7) and (8), $\frac{d V(x(t))}{d t}$ can be evaluated as follows:

$$
\begin{aligned}
\dot{V} & =\left(A_{F} x+B_{F} e\right)^{\mathrm{T}} P(\varepsilon) x+x^{\mathrm{T}} P(\varepsilon)\left(A_{F} x+B_{F} e\right) \\
& =x^{\mathrm{T}}\left(A^{\mathrm{T}} P(\varepsilon)+P(\varepsilon) A-2 P(\varepsilon) B B^{\mathrm{T}} P(\varepsilon)\right) x-2 x^{\mathrm{T}} K^{\mathrm{T}} K e \\
& =f_{1}(t)-(1-\sigma) x(t)^{\mathrm{T}} Q(\varepsilon) x(t)-(1-\sigma) x(t)^{\mathrm{T}} K^{\mathrm{T}} K x(t) .
\end{aligned}
$$

If $t_{k+1}>t_{k}+\tau, k \geq 0$, the event-trigger strategy in Algorithm 1 enforces that $f(t) \leq 0, \forall t \in$ $\left[t_{k}+\tau, t_{k+1}\right]$. Then, the following claim is straightforward.

Claim 1: For any possible $t \in\left(t_{k}+\tau, t_{k+1}\right]$, if $\left\|u\left(t_{k}\right)\right\|_{\infty} \leq \varpi$, then

$$
\dot{V} \leq-(1-\sigma) x(t)^{\mathrm{T}} Q(\varepsilon) x(t)-(1-\sigma) x(t)^{\mathrm{T}} K^{\mathrm{T}} K x(t) \leq 0 .
$$

\section{Step 2. Bounded Control.}

Define the desirable control $\tilde{u}(t)$ as

$$
\tilde{u}(t) \triangleq-K x(t) .
$$

One has $\tilde{u}\left(t_{k}\right)=u\left(t_{k}\right)$ and $u\left(t_{k+1}\right)=\tilde{u}\left(t_{k+1}\right)$. By Lemma 2 , one obtains

$$
\|\tilde{u}\|_{\infty}^{2} \leq\|K x\|^{2} \leq\left\|B^{\mathrm{T}} P(\varepsilon) B\right\| \cdot V(x) .
$$

Denote

$$
\beta(\varepsilon) \triangleq \frac{1}{\left\|B^{\mathrm{T}} P(\varepsilon) B\right\|} .
$$

For brevity, $\beta(\varepsilon)$ is denoted as $\beta$ in the sequel. Then, the following claim is straightforward.

Claim 2: For any $t \in\left[t_{k}, t_{k+1}\right)$, if $V(x(t)) \leq \beta \varpi^{2}$, then $\|\tilde{u}(t)\|_{\infty} \leq \varpi$.

Step 3. Design of $\tau$.

A lower bound $\tau$ of the event-triggering time interval will first be designed using the low-gain parameter $\varepsilon$ and an auxiliary parameter $\delta \in(0,1)$, which are to be determined later. Denote

$$
a_{0}=\left\|A_{F}\right\|, \quad a_{1}=\left\|A_{F}\right\|+\left\|B_{F}\right\|, \quad a_{2}=\left\|B_{F}\right\|,
$$

where $A_{F}=A-B K(\varepsilon)$ and $B_{F}=-B K(\varepsilon)$. By Lemma 1 and Assumption $1, A_{F}$ is Hurwitz, $a_{0}>0 ; B_{F} \neq 0, a_{1}>a_{2}>0$; and $a_{1}^{2} \geq 4 a_{2} a_{0}$. Using Lemma 8 in Appendix A with $\tilde{t}=t_{k}$, 
if $\mu=\left|a_{0}-a_{2}\right|=\sqrt{a_{1}^{2}-4 a_{2} a_{0}}>0$, by $(\mathrm{A} 1), \phi(t)=b_{1}\left(1-e^{-\mu\left(t-t_{k}\right)}\right) /\left(e^{-\mu\left(t-t_{k}\right)}-b_{2}\right)$, where $b_{1}=\left(a_{1}-\mu\right) /\left(2 a_{2}\right)$ and $b_{2}=\left(a_{1}-\mu\right) /\left(a_{1}+\mu\right)$. Now, using $\varepsilon$ and $\delta$, the minimum inter-event time $\tau$ is designed as $\tau(\varepsilon, \theta)$ for Algorithm 1:

$$
\tau(\varepsilon, \delta)=\frac{1}{\mu} \ln \frac{2 a_{0}+\left(a_{1}+\mu\right) \delta}{2 a_{0}+\left(a_{1}-\mu\right) \delta}>0
$$

so that $\phi\left(t_{k}+\tau(\varepsilon, \delta)\right)=\delta$. If $\mu=\left|a_{0}-a_{2}\right|=0$, then $\phi(t)=\frac{1}{a_{2}\left(\frac{2}{a_{1}}+t_{k}-t\right)}-\frac{a_{1}}{2 a_{2}}$, one can let

$$
\tau(\varepsilon, \delta)=\frac{2}{a_{1}}-\frac{2}{a_{1}+2 a_{2} \delta}=\frac{\delta}{(1+\delta) a_{2}}
$$

so that $\phi\left(t_{k}+\tau(\varepsilon, \delta)\right)=\delta$. For brevity, $\tau(\varepsilon, \delta)$ is denoted as $\tau$ in the sequel. The proof of the following claim is given in Appendix B.

Claim 3: If $\left\|u\left(t_{k}\right)\right\|_{\infty} \leq \varpi$ and $\|x(t)\|$ is positive for all $t \in\left[t_{k}, t_{k}+\tau\right)$, then

$$
\|e(t)\| \leq \delta\|x(t)\|
$$

holds on $\left[t_{k}, t_{k}+\tau\right]$.

For any $\delta \in(0,1)$ satisfying that

$$
\Upsilon(\varepsilon, \delta) \triangleq Q(\varepsilon)-\delta^{2}\|K\|^{2} I_{n} \succ 0
$$

by (9), if (16) holds and $\left\|u\left(t_{k}\right)\right\|_{\infty} \leq \varpi$, using Lemma 2 and the fact that

$$
\gamma a^{\mathrm{T}} Y a+\frac{1}{\gamma} b^{\mathrm{T}} Y b \geq \pm 2 a^{\mathrm{T}} Y b
$$

for any $Y \succeq 0$ and $\gamma>0$, one has

$$
\begin{aligned}
\dot{V} & \leq-x^{\mathrm{T}} Q(\varepsilon) x+e^{\mathrm{T}} K^{\mathrm{T}} K e \\
& \leq-x^{\mathrm{T}}\left[Q(\varepsilon)-\delta^{2}\|K\|^{2} I_{n}\right] x \\
& =-x(t)^{\mathrm{T}} \Upsilon(\varepsilon, \delta) x(t) \leq 0 .
\end{aligned}
$$

Claims 2, 3 together with Claim 4 in the following step will be used to establish that if $x\left(t_{k}\right) \neq 0$, $k \geq 0$, then for all $t \in\left[t_{k}, t_{k}+\tau\right]$, (i) $\|x(t)\|>0$; (ii) $\|e(t)\| \leq \delta\|x(t)\|$; (iii) $\|\tilde{u}(t)\|_{\infty} \leq \varpi$. The rationale for this procedure is: (i) utilizing the event-triggering conditions; (ii) using the fact that $e\left(t_{k}\right)=0$; (iii) setting the low-gain parameter such that $V(x(0)) \leq \beta \varpi^{2}$.

\section{Step 4. Design of Parameters.}

Noting the boundedness of $\mathcal{X}$ and the fact that $\lim _{\varepsilon \rightarrow 0^{+}} P(\varepsilon)=0$, established in Lemma 1, one can set the low-gain parameter as $\varepsilon=\varepsilon^{*}$ such that

$$
\left\|B^{\mathrm{T}} P\left(\varepsilon^{*}\right) B\right\| \cdot \sup _{x \in \mathcal{X}} x^{\mathrm{T}} P\left(\varepsilon^{*}\right) x \leq \varpi^{2} .
$$

By Claim 2 and (12), one has

$$
V(x(0)) \leq \beta \varpi^{2} \text { and }\|u(0)\|_{\infty} \leq \varpi
$$


for any initial values $x(0) \in \mathcal{X}$.

Next, using (17), $\delta$ is set as $\delta=\delta^{*} \in(0,1)$ such that

$$
\Upsilon\left(\varepsilon^{*}, \delta^{*}\right) \succ 0 .
$$

The proof of the following claim is given in Appendix B.

Claim 4: Let $\varepsilon=\varepsilon^{*}$ and $\delta=\delta^{*}$ as specified in (20) and (22). If $x\left(t_{k}\right) \neq 0$ and $V\left(x\left(t_{k}\right)\right) \leq \beta \varpi^{2}$, then $\|x(t)\|>0, V(x(t)) \leq \beta \varpi^{2},(16)$ holds, and $\|\tilde{u}(t)\|_{\infty} \leq \varpi, \forall t \in\left[t_{k}, t_{k}+\tau\right]$.

\section{Step 5. Exponential Stability.}

If $x(0) \neq 0$, using (19), (21), and Claims 1, 2, and 4, one has that $\dot{V} \leq 0$ and $V\left(x\left(t_{1}\right)\right)<V(x(0))$. If $x(t) \neq 0, \forall t \leq t_{k}$, then $\dot{V}<0, \forall t \leq t_{k}$, and $V\left(x\left(t_{k}\right)\right)<\beta \varpi^{2}$. Using (19) and Claims 1, 2, and 4, one obtains that

$$
x(t) \neq 0, \dot{V}<0, \forall t<t_{k+1} \text {, and } V\left(x\left(t_{k+1}\right)\right)<\beta \varpi^{2} .
$$

Therefore, if $x(t)=0$ is achieved in a finite time, $t=t_{k+1}$ is triggered by $f_{2}\left(t_{k+1}\right)=0$. Then, for all $t \geq t_{k+1}$, one has $u=0$, and $x(t) \equiv 0$.

If $x(t) \neq 0, \forall t \geq 0$, using (19) and Claims 1,2, and 4, one has that either (10) or (19) holds for $t \in\left(t_{k}, t_{k+1}\right)$, and $V_{+}^{\prime}<0$ at $t=t_{k}, \forall k \geq 0$. Thus, by Lyapunov stability theory (Michel et al., 2008), one obtains the exponential convergence $\lim _{t \rightarrow \infty}\|x(t)\|=0$.

Remark 2: The low-gain parameter is designed as $\varepsilon=\varepsilon^{*}$ specified in (20) and the lower bound $\tau$ of the event-triggering time interval is designed as $\tau(\varepsilon, \delta)$ given in (14) with $\varepsilon=\varepsilon^{*}$ and $\delta=\delta^{*}$, where $\delta^{*}$ is set in (22). To find an $\varepsilon^{*}$ fulfilling (20), the method of bisection (Su et al., 2014) may be applied. For the event-trigger parameters $\sigma$ and $\theta$, if $\sigma$ and $\theta$ are too small, control updates will be very frequent. Therefore, one needs to select the values of $\sigma$ and $\theta$ appropriately. In addition, if the control performance is taken into account, the parameter-dependent matrix $Q(\varepsilon)$ can be designed, and the term $P(\varepsilon) B B^{\mathrm{T}} P(\varepsilon)$ in ARE (3) can be substituted by $P(\varepsilon) B R(\varepsilon)^{-1} B^{\mathrm{T}} P(\varepsilon)$ with the parameter-dependent matrix $R(\varepsilon)$ to be designed.

Remark 3: If the bounded set $\mathcal{X}$, within which the initial value $x(0)$ is known to be located, is $\mathcal{X}=\left\{x \in \mathbb{R}^{n} \mid\|x\| \leq R_{\mathcal{X}}\right\}$ with $R_{\mathcal{X}}>0$, then condition (20) is satisfied by setting $\varepsilon^{*}$ such that

$$
\left\|B^{\mathrm{T}} P\left(\varepsilon^{*}\right) B\right\| \cdot\left\|P\left(\varepsilon^{*}\right)\right\|<\frac{\varpi^{2}}{\left(R_{\mathcal{X}}\right)^{2}},
$$

which can easily be numerically checked when solving ARE (3). One can note that if $R_{\mathcal{X}}$ is large, the low-gain parameter $\varepsilon^{*}$ needs to be very small, which will result in slow convergence. To improve the performance, the high-low gain design is proposed in next section.

Remark 4: The idea of the a priori setting of the inactive interval $\left[t_{k}, t_{k}+\tau\right)$, as well as the proof of Claim 3 in Appendix B.1 based on the Riccati ODE, is similar to the self-triggered control in (Anta \& Tabuada, 2010; Wang \& Lemmon, 2009). A difference is that the self-triggered control is to reduce the cost of continuous-time monitoring of the state; while in this paper, the necessity of setting an inactive interval $\left[t_{k}, t_{k}+\tau\right)$ is mainly due to the difficulty in providing an inherent minimum inter-event time for the proposed ETC algorithms based on the event-triggering function (5). 


\subsection{High-low gain design}

The low-gain design can be improved by scheduling the controller using the high-low gain techniques (Grognard et al. (2002); Lin (1999)). For brevity, only the single-input systems are considered, that is, $B \in \mathbb{R}^{n}$.

\section{Algorithm 3: Event-based updating:}

Step 1. If $x(0) \neq 0$, set $t_{0} \triangleq 0$; otherwise, both the controller and the event trigger are inactive. At the beginning of each updating process, $t=t_{k}, k \geq 0$, the feedback control input $u$ is updated. A minimum inter-event time $\tau_{k}>0$, which will be designed in Section 3.3, is set a priori. During the time interval $\left[t_{k}, t_{k}+\tau_{k}\right)$, the event trigger is inactive.

Step 2. At the time $t=t_{k}+\tau_{k}$, the event trigger is activated. An event-triggering function $f(t)$ satisfying $f\left(t_{k}\right) \leq 0$ will be designed in Section 3.2. Either if $f\left(t_{k}+\tau_{k}\right)>0$, or if $f\left(t_{k}+\tau_{k}\right)=0$ while $\left\|x\left(t_{k}+\tau_{k}\right)\right\| \neq 0$, the next event is triggered at $t_{k+1}=t_{k}+\tau_{k}$; if $f\left(t_{k}+\tau_{k}\right)<0$, for $t>t_{k}+\tau_{k}$, the next updating event is triggered at instant $t_{k+1}>t_{k}+\tau_{k}$ when $f\left(t_{k+1}\right)=0$ and $f(t)<0$ for all $t \in\left[t_{k}+\tau_{k}, t_{k+1}\right)$; if $f\left(t_{k}+\tau_{k}\right)=\left\|x\left(t_{k}+\tau_{k}\right)\right\|=0$, stabilization has been achieved in a finite time; if $f(t)<0$ for all $t \in\left[t_{k}+\tau_{k},+\infty\right)$, denote $t_{k+1} \triangleq+\infty$. The feedback control will be designed in the linear form of $u(t)=-K x\left(t_{k}\right), t \in\left[t_{k}, t_{k+1}\right)$.

Step 3. When a finite $t_{k+1}$ is triggered, a new updating cycle will begin, then go to Step 1.

The following lemma is important for the high-low gain design.

Lemma 3: Let Assumption 1 hold, $B \in \mathbb{R}^{n}, r>0$, and $Q(\varepsilon) \succ 0, \lim _{\varepsilon \rightarrow 0^{+}} Q(\varepsilon)=0$, where $\varepsilon>0$ is a low-gain parameter. For each $\varepsilon>0$, there exists a unique positive definite matrix $P=P(\varepsilon)$ that solves the ARE

$$
A^{\mathrm{T}} P(\varepsilon)+P(\varepsilon) A-\frac{1}{r} P(\varepsilon) B B^{\mathrm{T}} P(\varepsilon)+Q(\varepsilon)=0 .
$$

Moreover, $\left(A-\frac{\alpha}{r} B B^{\mathrm{T}} P\right)$ is Hurwitz for any $\alpha \geq \frac{1}{2}$; and $\lim _{\varepsilon \rightarrow 0^{+}} P(\varepsilon)=0$.

Proof. By Lemma 1, it suffices to prove the Hurwitz stability of $A_{F} \triangleq A-\alpha B r^{-1} B^{\mathrm{T}} P$. It is straightforward that for any $\alpha \geq \frac{1}{2}$,

$$
-A_{F}^{\mathrm{T}} P-P A_{F}=(2 \alpha-1) P B r^{-1} B^{\mathrm{T}} P+Q(\varepsilon) \succ 0 .
$$

By the property of the Lyapunov equation, one has that $A_{F}$ is Hurwitz.

Let $P=P(\varepsilon) \succ 0$ be the solution to ARE (24). Denote

$$
\mathcal{T}(\varepsilon) \triangleq\left\{x \in \mathbb{R}^{n} \mid x^{\mathrm{T}} P(\varepsilon) x \leq \frac{r^{2} \varpi^{2}}{B^{\mathrm{T}} P(\varepsilon) B}\right\},
$$

where $\varpi$ is the input saturation threshold. Similar to (Grognard et al., 2002, Eq. (3)), one has that for any $x$ located within the set $\mathcal{T}(\varepsilon),\left|B^{\mathrm{T}} P(\varepsilon) x\right| \leq r \varpi$.

The high-low gain design of a bounded control protocol for continuous-time system (1) is performed in three steps.

Algorithm 4: Event-based high-low gain stabilization: 
Step 1. For any initial state $x_{0}$, the low-gain parameter is set as $\varepsilon=\varepsilon_{0}$ :

$$
\varepsilon_{0} \triangleq \max \left\{\varepsilon \in(0,1]: x_{0}^{\mathrm{T}} P(\varepsilon) x_{0} \cdot B^{\mathrm{T}} P(\varepsilon) B \leq r^{2} \varpi^{2}\right\} \text {. }
$$

Find $P_{0} \triangleq P\left(\varepsilon_{0}\right) \succ 0$ to solve $\operatorname{ARE}(24)$ with $\varepsilon=\varepsilon_{0}$. Meanwhile, denote $\varepsilon_{1} \triangleq 1$, and find $P_{1} \triangleq P(1) \succ 0$ to solve $\operatorname{ARE~(24)~with~} \varepsilon=\varepsilon_{1}=1$. Denote

$$
K_{0} \triangleq r^{-1} B^{\mathrm{T}} P_{0}, \quad K_{1} \triangleq r^{-1} B^{\mathrm{T}} P_{1} .
$$

Using (25), denote

$$
\mathcal{T}_{1} \triangleq \mathcal{T}(1), \quad \mathcal{T}_{0} \triangleq \mathbb{R}^{n} \backslash \mathcal{T}_{1}
$$

Step 2. For $x\left(t_{k}\right) \in \mathcal{T}_{i}$, a high-gain parameter $\alpha(t)$ is defined:

$$
\alpha\left(t_{k}\right) \triangleq \max \left\{\alpha \in\left[1, \frac{1}{\varepsilon_{i}}\right]: \alpha \cdot \sqrt{x\left(t_{k}\right)^{\mathrm{T}} P_{i} x\left(t_{k}\right)} \leq \frac{r \varpi}{\sqrt{B^{\mathrm{T}} P_{i} B}}\right\} .
$$

For the event-trigger parameters $\sigma, \theta \in(0,1)$ that are appropriately selected, denote

$$
\begin{aligned}
& f_{1}(t)=-\sigma x(t)^{\mathrm{T}} Q\left(\varepsilon_{i}\right) x(t)-\sigma\left(2 \alpha\left(t_{k}\right)-1\right) \cdot x(t)^{\mathrm{T}} K_{i}^{\mathrm{T}} r K_{i} x(t)-2 \alpha\left(t_{k}\right) \cdot x(t)^{\mathrm{T}} K_{i}^{\mathrm{T}} r K_{i} e(t), \\
& f_{2}(t)=\left\|K_{i} e(t)\right\|-\theta\left\|K_{i} x(t)\right\| .
\end{aligned}
$$

The event-triggering function is designed as follows:

$$
f(t)= \begin{cases}f_{1}(t), & \text { if } x\left(t_{k}\right) \in \mathcal{T}_{0} \\ \max \left\{f_{1}(t), f_{2}(t)\right\}, & \text { if } x\left(t_{k}\right) \in \mathcal{T}_{1}\end{cases}
$$

Step 3. A feedback law using $x\left(t_{k}\right) \in \mathcal{T}_{i}$ is designed as

$$
u(t)=-\alpha\left(t_{k}\right) K_{i} x\left(t_{k}\right), t \in\left[t_{k}, t_{k+1}\right), k \geq 0 .
$$

Remark 5: The high-gain scheduling by the parameter $\alpha(t)$ is stopped once the state $x\left(t_{k}\right)$ reaches the set $\mathcal{T}_{1}$. As suggested in (Grognard et al. (2002); Lin (1999)), a heuristic scheduling rule is to let $\alpha$ be proportional to $1 / \varepsilon$. The ETC controller scheduling in the high-low gain design only relies on the online adaptation of the scalar control gain $\alpha(t)$. In the traditional high-low gain design of bounded control, the controller scheduling involves in a complicated adaptation of the low-gain parameter $\varepsilon$, which cannot be easily applied to the design of the event-triggered control protocol.

Similar to Lemma 2, the following lemma holds.

Lemma 4: The controller matrices $K_{i}$ in (31) satisfy that $r^{-1} B^{\mathrm{T}} P_{i} B \cdot P_{i} \succeq K_{i}^{\mathrm{T}} r K_{i}$.

Proof. It is straightforward that $r^{-1} B^{\mathrm{T}} P_{i} B=\left\|P_{i}^{\frac{1}{2}} B r^{-1} B^{\mathrm{T}} P_{i}^{\frac{1}{2}}\right\|$. Then, one has $r^{-1} B^{\mathrm{T}} P_{i} B \cdot P_{i} \succeq$ $P_{i}^{\frac{1}{2}}\left(P_{i}^{\frac{1}{2}} B r^{-1} B^{\mathrm{T}} P_{i}^{\frac{1}{2}}\right) P_{i}^{\frac{1}{2}}=K_{i}^{\mathrm{T}} r K_{i}$.

Theorem 2: Consider continuous-time system (1) subject to actuator saturation with saturation threshold $\varpi>0$. Let Assumption 1 hold and $B \in \mathbb{R}^{n}$. Then, Algorithms 3 and 4 can achieve global exponential stabilization of system (1). That is, for any initial state $x(0) \in \mathbb{R}^{n}$, and any 
$\sigma, \theta \in(0,1)$ in the event-triggering function (30), there exists a sequence $\left\{\tau_{k}\right\}$ for Algorithm 3, such that $\lim _{t \rightarrow \infty} x(t)=0$ is achieved exponentially. Furthermore, $\tau_{k} \geq \tau_{\min }$ for some $\tau_{\min }>0$.

Proof. Step 1. Attractivity of $\mathcal{T}_{1}$.

Assume that $x\left(t_{0}\right)$ is located in $\mathcal{T}_{0}$. Denote

$$
\alpha(t)=\alpha\left(t_{k}\right), t \in\left[t_{k}, t_{k+1}\right), k \geq 0 .
$$

Similar to (7), when saturation does not occur and $x\left(t_{k}\right)$ is located within $\mathcal{T}_{0}$, the closed-loop dynamics will be

$$
\dot{x}(t)=A_{F} x(t)+B_{F} e(t), \dot{e}(t)=-A_{F} x(t)-B_{F} e(t),
$$

where $A_{F} \triangleq A-\alpha(t) B K_{0}$ and $B_{F} \triangleq-\alpha(t) B K_{0}$.

Before the state $x\left(t_{k}\right)$ reaches $\mathcal{T}_{1}$, the following quadratic Lyapunov function is used:

$$
V_{0}(x(t)) \triangleq x(t)^{\mathrm{T}} P_{0} x(t)
$$

When saturation does not occur, $\frac{d V_{0}(x(t))}{d t}$ can be evaluated as follows:

$$
\begin{aligned}
\dot{V}_{0} & =x^{\mathrm{T}}\left(A^{\mathrm{T}} P_{0}+P_{0} A-2 \alpha P_{0} B r^{-1} B^{\mathrm{T}} P_{0}\right) x-2 \alpha x^{\mathrm{T}} K_{0}^{\mathrm{T}} r K_{0} e \\
& =f_{1}(t)-(1-\sigma) x(t)^{\mathrm{T}} Q\left(\varepsilon_{0}\right) x(t)-(2 \alpha-1) \cdot(1-\sigma) x(t)^{\mathrm{T}} K_{0}^{\mathrm{T}} r K_{0} x(t) .
\end{aligned}
$$

Define the desirable control $\tilde{u}(t)$ as

$$
\tilde{u}(t) \triangleq-\alpha(t) K_{0} x(t)
$$

By Lemma 4 , one has $\|\tilde{u}\|_{\infty}^{2} \leq \alpha^{2} r^{-2} B^{\mathrm{T}} P_{0} B \cdot V_{0}(x)$. Denote

$$
\beta_{0} \triangleq \frac{r^{2}}{B^{\mathrm{T}} P_{0} B}
$$

For any $t \in\left[t_{k}, t_{k+1}\right)$, if $V_{0}(x(t)) \leq \frac{\beta_{0} \varpi^{2}}{\alpha\left(t_{k}\right)^{2}}$, then $\|\tilde{u}(t)\|_{\infty} \leq \varpi$.

Denote

$$
\delta_{k} \triangleq \frac{0.95 \sqrt{\lambda_{\min }\left(Q\left(\varepsilon_{0}\right)\right) \cdot\left(2 \alpha\left(t_{k}\right)-1\right)}}{\alpha\left(t_{k}\right) \sqrt{r}\left\|K_{0}\right\|}
$$

such that

$$
\Upsilon\left(\delta_{k}\right) \triangleq Q\left(\varepsilon_{0}\right)-\frac{\alpha\left(t_{k}\right)^{2}}{2 \alpha\left(t_{k}\right)-1} \delta_{k}^{2} r\left\|K_{0}\right\|^{2} I_{n} \succ 0 .
$$

Denote $a_{0}=\left\|A_{F}\right\|, a_{1}=\left\|A_{F}\right\|+\left\|B_{F}\right\|, a_{2}=\left\|B_{F}\right\|$, where $A_{F}=A-\alpha\left(t_{k}\right) B K_{0}$ and $B_{F}=$ $-\alpha\left(t_{k}\right) B K_{0}$. Using Lemma 8 in Appendix A with $\tilde{t}=t_{k}$, if $\mu=\left|a_{0}-a_{2}\right|>0$, the minimum inter-event time $\tau_{k}$ for Algorithm 3 is designed as:

$$
\tau_{k}=\frac{1}{\mu} \ln \frac{2 a_{0}+\left(a_{1}+\mu\right) \delta_{k}}{2 a_{0}+\left(a_{1}-\mu\right) \delta_{k}}>0
$$


so that $\phi\left(t_{k}+\tau_{k}\right)=\delta_{k}$. If $\mu=\left|a_{0}-a_{2}\right|=0$, then define

$$
\tau_{k}=\frac{\delta_{k}}{\left(1+\delta_{k}\right)\left\|B K_{0}\right\| \cdot \alpha\left(t_{k}\right)}
$$

so that $\phi\left(t_{k}+\tau_{k}\right)=\delta_{k}$. Similar to Claim 3 in the proof of Theorem 1, if $\left\|u\left(t_{k}\right)\right\|_{\infty} \leq \varpi$ and $\|x(t)\|$ is positive for all $t \in\left[t_{k}, t_{k}+\tau_{k}\right)$, then

$$
\|e(t)\| \leq \delta_{k}\|x(t)\|
$$

holds on $\left[t_{k}, t_{k}+\tau_{k}\right]$.

If (40) holds and $\left\|u\left(t_{k}\right)\right\|_{\infty} \leq \varpi$, denoting $\gamma_{k} \triangleq \frac{2 \alpha\left(t_{k}\right)-1}{\alpha\left(t_{k}\right)}$ and using (18), one has

$$
\begin{aligned}
\dot{V}_{0} & \leq-x^{\mathrm{T}} Q\left(\varepsilon_{0}\right) x-(2 \alpha-1) x^{\mathrm{T}} K_{0}^{\mathrm{T}} r K_{0} x+\alpha \gamma_{k} x^{\mathrm{T}} K_{0}^{\mathrm{T}} r K_{0} x+\frac{\alpha}{\gamma_{k}} e^{\mathrm{T}} K_{0}^{\mathrm{T}} r K_{0} e \\
& \leq-x^{\mathrm{T}}\left[Q\left(\varepsilon_{0}\right)-\frac{\alpha\left(t_{k}\right)^{2}}{2 \alpha\left(t_{k}\right)-1} \delta_{k}^{2} r\left\|K_{0}\right\|^{2} I_{n}\right] x \\
& =-x(t)^{\mathrm{T}} \Upsilon\left(\delta_{k}\right) x(t) \leq 0 .
\end{aligned}
$$

Then, similar to Claim 4 in the proof of Theorem 1 , if $x\left(t_{k}\right) \neq 0$ and $V_{0}\left(x\left(t_{k}\right)\right) \leq \frac{\beta_{0} \varpi^{2}}{\alpha\left(t_{k}\right)^{2}}$, then $\|x(t)\|>0, V_{0}(x(t)) \leq \frac{\beta_{0} \varpi^{2}}{\alpha\left(t_{k}\right)^{2}},(40)$ holds, and $\|\tilde{u}(t)\|_{\infty} \leq \varpi, \forall t \in\left[t_{k}, t_{k}+\tau\right]$.

Because $x(0) \in \mathcal{T}_{0}$, one has $x(0) \neq 0$, and $V_{0}\left(x\left(t_{1}\right)\right)<V_{0}(x(0))$. Before $x\left(t_{k}\right)$ reaches $\mathcal{T}_{1}$, by $(34)$ and (41), $V_{0}(x(t))$ decreases exponentially. Therefore, $x\left(t_{k}\right)$ will reach $\mathcal{T}_{1}$ in a finite time.

Step 2. Local stability within $\mathcal{T}_{1}$.

Assume that $x\left(t_{k}\right)$ reaches $\mathcal{T}_{1}$ at the event time $t_{k}=t_{\tilde{k}}$. Consider the following quadratic Lyapunov function:

$$
V_{1}(x(t)) \triangleq x(t)^{\mathrm{T}} P_{1} x(t)
$$

By (25) and (28), one has

$$
V_{1}\left(x\left(t_{\tilde{k}}\right)\right) \leq \frac{r^{2} \varpi^{2}}{B^{\mathrm{T}} P_{1} B} .
$$

For any $\forall k \geq \tilde{k}$, let $\alpha\left(t_{k}\right)=1$, and

$$
\delta_{k}=\tilde{\delta}=\frac{0.95 \sqrt{\lambda_{\min }(Q(1))}}{\sqrt{r}\left\|K_{1}\right\|}
$$

such that

$$
\Upsilon(\tilde{\delta}) \triangleq Q(1)-\tilde{\delta}^{2} r\left\|K_{1}\right\|^{2} I_{n} \succ 0 .
$$

Denote $a_{0}=\left\|A-B K_{1}\right\|, a_{2}=\left\|B K_{1}\right\|, a_{1}=a_{0}+a_{2}$. Using Lemma 8 in Appendix A, if $\mu=$ $\left|a_{0}-a_{2}\right|>0$, the minimum inter-event time $\tau_{k}$ for Algorithm 3 is designed as:

$$
\tau_{k}=\tilde{\tau}=\frac{1}{\mu} \ln \frac{2 a_{0}+\left(a_{1}+\mu\right) \tilde{\delta}}{2 a_{0}+\left(a_{1}-\mu\right) \tilde{\delta}}>0
$$


so that $\phi\left(t_{k}+\tilde{\tau}\right)=\tilde{\delta}, \forall k \geq \tilde{k}$. If $\mu=\left|a_{0}-a_{2}\right|=0$, then let

$$
\tau_{k}=\tilde{\tau}=\frac{\tilde{\delta}}{(1+\tilde{\delta})\left\|B K_{1}\right\|}
$$

so that $\phi\left(t_{k}+\tilde{\tau}\right)=\tilde{\delta}, \forall k \geq \tilde{k}$. Similar to the proof of Theorem 1 , one has that the set $\mathcal{T}_{1}$ is invariant as $V_{1}(x(t))$ is non-increasing; and the exponential convergence $\lim _{t \rightarrow \infty}\|x(t)\|=0$ is obtained. Because $\tau_{k} \equiv \tilde{\tau}, \forall k \geq \tilde{k}$, it is obvious that the sequence $\left\{\tau_{k}\right\}$ is lower-bounded by a positive $\tau_{\min }$.

\section{Discrete-time Systems}

In this section, system (1) is treated as a discrete-time system subject to actuator saturation. A discrete-time event-trigger strategy is proposed in Section 4.1. A bounded control protocol is designed in Section 4.2. The semi-global stabilization result is established in Section 4.3. The high-low gain design for global stabilization is studied in Section 4.4.

\subsection{Event-trigger Strategy}

In this subsection, the event-triggered mechanism is described to generate the updating time sequence $\left\{t_{k}\right\}$ for discrete-time systems.

\section{Algorithm 5: Event-based updating:}

Step 1. The initial time is set as the first event time: $t_{0} \triangleq 0$. At the beginning of each updating process, $t=t_{k}, k \geq 0$, the feedback control input $u$ is updated. Design an event-triggering function $f(t)$ such that $f\left(t_{k}\right) \leq 0$.

Step 2. For $t \geq t_{k}+1$, the next updating event is triggered at instant $t_{k+1} \geq t_{k}+1$ when $f\left(t_{k+1}\right)>0$ and $f(t) \leq 0$ for $t<t_{k+1}$. If no such an event occurs, denote $t_{k+1} \triangleq+\infty$.

Step 3. When a finite $t_{k+1}$ is triggered, a new updating cycle will begin, then go to Step 1 and redefine the event-triggering function $f(t)$ such that $f\left(t_{k+1}\right) \leq 0$.

\subsection{Bounded Control Protocol}

The following assumption plays an important role in the discrete-time stabilization through bounded control.

Assumption 2: The pair $(A, B)$ is asymptotically null controllable under bounded control; that is, (i) the pair $(A, B)$ is stabilizable; (ii) $\rho(A) \leq 1$. In addition, since control is unnecessary for stabilization when $A$ is Schur, it is assumed that $A$ is not Schur and $B \neq 0$.

Lemma 5: (Lin, 1999) Let Assumption 2 hold, $R \succ 0$, and $Q(\varepsilon)$ be a parameter-dependent positive definite matrix satisfying the monotonic convergence $\lim _{\varepsilon \rightarrow 0^{+}} Q(\varepsilon)=0$. Then, for each $\varepsilon>0$, there exists a unique positive definite matrix $P=P(\varepsilon)$ that solves the discrete-time ARE (DARE)

$$
P=A^{\mathrm{T}} P A-A^{\mathrm{T}} P B\left(B^{\mathrm{T}} P B+R\right)^{-1} B^{\mathrm{T}} P A+Q(\varepsilon) .
$$

Moreover, $\left(A-B\left(B^{\mathrm{T}} P B+R\right)^{-1} B^{\mathrm{T}} P A\right)$ is Schur; and $\lim _{\varepsilon \rightarrow 0^{+}} P(\varepsilon)=0$. 
The design of a bounded control protocol for discrete-time system (1) is performed in three steps. Algorithm 6: Event-based low-gain stabilization:

Step 1. Find $P=P(\varepsilon) \succ 0$ to solve the DARE

$$
P=A^{\mathrm{T}} P A-A^{\mathrm{T}} P B\left(B^{\mathrm{T}} P B+I_{n}\right)^{-1} B^{\mathrm{T}} P A+Q(\varepsilon),
$$

where $\varepsilon>0$ is a low-gain parameter to be designed and $Q(\varepsilon)$ is a parameter-dependent positive definite matrix satisfying the monotonic convergence $\lim _{\varepsilon \rightarrow 0^{+}} Q(\varepsilon)=0$.

Step 2. Denote that

$$
K(\varepsilon) \triangleq\left(B^{\mathrm{T}} P(\varepsilon) B+I_{n}\right)^{-1} B^{\mathrm{T}} P(\varepsilon) A .
$$

For brevity, $P(\varepsilon)$ and $K(\varepsilon)$ are denoted as $P$ and $K$, respectively in the sequel. By Lemma 5 and Assumption 2, one has that $(A-B K)$ is Schur and $B K \neq 0$. Then, the event-triggering function is designed as

$$
\begin{aligned}
& f(t)=\max \left\{f_{1}(t), f_{2}(t)\right\}, \\
& f_{1}(t) \triangleq-\sigma x(t)^{\mathrm{T}} Q(\varepsilon) x(t)-\sigma x(t)^{\mathrm{T}} K^{\mathrm{T}} K x(t)+e(t)^{\mathrm{T}} K^{\mathrm{T}} B^{\mathrm{T}} P B K e(t)-2 x(t)^{\mathrm{T}} K^{\mathrm{T}} K e(t), \\
& f_{2}(t) \triangleq\|K e(t)\|-\theta\|K x(t)\|,
\end{aligned}
$$

where the event-trigger parameters $\sigma, \theta \in(0,1)$ are appropriately selected.

Step 3. A feedback law using $x\left(t_{k}\right)$ is designed as

$$
u(t)=-K x\left(t_{k}\right), t_{k} \leq t<t_{k+1}, k \geq 0 .
$$

Lemma 6: The unique positive definite solution $P$ to DARE (46) and the controller matrix $K$ in (47) satisfy that

$$
P-Q(\varepsilon) \succeq K^{\mathrm{T}} K=A^{\mathrm{T}} P B K-K^{\mathrm{T}} B^{\mathrm{T}} P B K .
$$

Proof. It is straightforward to verify that $A^{\mathrm{T}} P B K=K^{\mathrm{T}}\left(B^{\mathrm{T}} P B+I_{n}\right) K=K^{\mathrm{T}} B^{\mathrm{T}} P B K+K^{\mathrm{T}} K$ and

$$
\begin{aligned}
P-Q(\varepsilon) & =A^{\mathrm{T}} P A-A^{\mathrm{T}} P B K=A_{F}^{\mathrm{T}} P A_{F}+K^{\mathrm{T}} K \\
& \succeq K^{\mathrm{T}} K, \quad A_{F} \triangleq A-B K .
\end{aligned}
$$

\subsection{Event-based semi-global stabilization}

The main discrete-time result for event-based semi-global stabilization is presented in the following theorem. 
Theorem 3: Consider discrete-time system (1) subject to actuator saturation with saturation threshold $\varpi>0$. Let Assumption 2 hold. Then, Algorithms 5 and 6 can achieve semi-global exponential stabilization of system (1). That is, for any bounded set $\mathcal{X}=\left\{x \in \mathbb{R}^{n} \mid\|x\| \leq R_{\mathcal{X}}\right\}$ with $R_{\mathcal{X}}>0$, and any $\sigma, \theta \in(0,1)$ in the event-triggering function (48), there exists a low-gain parameter $\varepsilon=\varepsilon^{*}$ such that $\lim _{t \rightarrow \infty} x(t)=0$ is achieved exponentially as long as $x(0) \in \mathcal{X}$.

Proof. Step 1. Closed-loop Dynamics.

By (1), (2) and (49), one has $u(t)=-K(e(t)+x(t))$. When saturation does not occur, the closed-loop dynamics will be

$$
x(t+1)=A_{F} x(t)+B_{F} e(t),
$$

where $A_{F} \triangleq A-B K$ and $B_{F} \triangleq-B K$.

For stability analysis, the following quadratic Lyapunov function is used:

$$
V(x(t)) \triangleq x^{\mathrm{T}} P x .
$$

By (46), (48), (52), and Lemma 6 , when $\|u\|_{\infty} \leq \varpi$, one can evaluate $\Delta V(t) \triangleq V(x(t+1))-$ $V(x(t))$, which is the variation of $V$ along the trajectory of $x(t)$, as follows:

$$
\begin{aligned}
\Delta V(t) & =x^{\mathrm{T}}\left(A_{F}^{\mathrm{T}} P A_{F}-P\right) x+e^{\mathrm{T}} K^{\mathrm{T}} B^{\mathrm{T}} P B K e-2 x^{\mathrm{T}} K^{\mathrm{T}} K e . \\
& =-x^{\mathrm{T}} Q(\varepsilon) x-x^{\mathrm{T}} K^{\mathrm{T}} K x+e^{\mathrm{T}} K^{\mathrm{T}} B^{\mathrm{T}} P B K e-2 x^{\mathrm{T}} K^{\mathrm{T}} K e \\
& =f_{1}(t)-(1-\sigma) x^{\mathrm{T}} Q(\varepsilon) x-(1-\sigma)\|K x\|^{2} .
\end{aligned}
$$

The event-trigger strategy in Algorithm 5 enforces $f_{1}(t) \leq 0$. By (53), if $\left\|u\left(t_{k}\right)\right\|_{\infty} \leq \varpi$, one has

$$
\Delta V(t) \leq-(1-\sigma) x^{\mathrm{T}} Q(\varepsilon) x-(1-\sigma)\|K x(t)\|^{2} \leq 0 .
$$

Step 2. Bounded Control.

Define the desirable control $\tilde{u}(t)$ as

$$
\tilde{u}(t) \triangleq-K x(t)
$$

Using (50), one obtains that

$$
\begin{aligned}
& \|\tilde{u}(t)\|_{\infty}^{2} \leq x^{\mathrm{T}} K^{\mathrm{T}} K x \leq V(x)-x^{\mathrm{T}} Q(\varepsilon) x \\
\leq & \eta(\varepsilon) V(x(t)), \quad \eta(\varepsilon) \triangleq 1-\frac{\lambda_{1}(Q(\varepsilon))}{\|P(\varepsilon)\|} .
\end{aligned}
$$

For brevity, $\eta(\varepsilon)$ is denoted as $\eta$ in the sequel. Noting that $K \neq 0$ and by (50), one has $\eta>0$. The following claim is straightforward.

Claim 5: If $V(x(t)) \leq \varpi^{2} / \eta$, then $\|\tilde{u}(t)\|_{\infty} \leq \varpi$.

Step 3. Design of Parameters.

By the fact that $\lim _{\varepsilon \rightarrow 0^{+}} P(\varepsilon)=0$, established in Lemma 5, the low-gain parameter $\varepsilon$ can be set 
as $\varepsilon=\varepsilon^{*}$ such that

$$
\sup _{x(0) \in \mathcal{X}} x(0)^{\mathrm{T}} P\left(\varepsilon^{*}\right) x(0) \cdot\left(1-\frac{\lambda_{1}\left(Q\left(\varepsilon^{*}\right)\right)}{\left\|P\left(\varepsilon^{*}\right)\right\|}\right) \leq \varpi^{2} .
$$

When $\mathcal{X}=\left\{x \in \mathbb{R}^{n} \mid\|x\| \leq R_{\mathcal{X}}\right\}$ with $R_{\mathcal{X}}>0$, condition (56) can be guaranteed by

$$
\left\|P\left(\varepsilon^{*}\right)\right\|-\lambda_{1}\left(Q\left(\varepsilon^{*}\right)\right) \leq \frac{\varpi^{2}}{\left(R_{\mathcal{X}}\right)^{2}}
$$

If (56) is satisfied, it is straightforward that $V(x(0)) \leq \varpi^{2} / \eta$ for any initial values $x(0) \in \mathcal{X}$. Using Claim 5 and (54), one obtains $\|u(0)\|_{\infty} \leq \varpi, V(x(t)) \leq \varpi^{2} / \eta,\|\tilde{u}(t)\|_{\infty} \leq \varpi$, and (54) holds for all $t \geq 0$, that is,

$$
\begin{aligned}
\Delta V(t) & \leq-(1-\sigma) x(t)^{\mathrm{T}} Q\left(\varepsilon^{*}\right) x(t)-(1-\sigma)\left\|K\left(\varepsilon^{*}\right) x(t)\right\|^{2} \\
& \leq-(1-\sigma) \lambda_{1}\left(Q\left(\varepsilon^{*}\right)\right)\|x(t)\|^{2} .
\end{aligned}
$$

By the Lyapunov stability theory, one obtains the exponential convergence $\lim _{t \rightarrow \infty}\|x(t)\|=0$. In addition, if the possible finite-time stabilization is achieved, that is, $x\left(t_{k}+s\right)=0$ for some $s \geq 0$, $k \geq 0$, then one has $f_{2}\left(t_{k}+s\right) \geq 0$. Thus, either $t_{k+1}=t_{k}+s$ if $f_{2}\left(t_{k}+s\right)>0$, or $t_{k+1}=+\infty$ if $K x\left(t_{k}\right)=0$. In both cases, one has that $x(t)=0, \forall t>t_{k}+s$, and the updating event will not be triggered thereafter.

\section{$4.4 \quad$ High-low gain design}

The low-gain design can be improved by scheduling the controller using the high-low gain techniques (Lin (1999)). For brevity, only the single-input systems are considered, that is, $B \in \mathbb{R}^{n}$. The following lemma is important for the high-low gain design.

Lemma 7: Let Assumption 2 hold, $B \in \mathbb{R}^{n}, Q(\varepsilon) \succ 0$ and $\lim _{\varepsilon \rightarrow 0^{+}} Q(\varepsilon)=0$. For each $\varepsilon>0$, the unique positive definite solution $P=P(\varepsilon)$ of the DARE

$$
P=A^{\mathrm{T}} P A-A^{\mathrm{T}} P B\left(B^{\mathrm{T}} P B+1\right)^{-1} B^{\mathrm{T}} P A+Q(\varepsilon)
$$

satisfies that $\left(A-\alpha B\left(B^{\mathrm{T}} P B+1\right)^{-1} B^{\mathrm{T}} P A\right)$ is Schur for $\alpha \in\left[\alpha_{1}(\varepsilon), \alpha_{2}(\varepsilon)\right]$, where

$$
\begin{aligned}
& \alpha_{2}(\varepsilon)=1+\frac{1}{B^{\mathrm{T}} P B}, \quad \frac{1}{2}<\alpha_{1}(\varepsilon)<1<\alpha_{2}(\varepsilon), \\
& \alpha_{1}(\varepsilon)=\alpha_{2}-\frac{\sqrt{B^{\mathrm{T}} P B+1}}{B^{\mathrm{T}} P B} .
\end{aligned}
$$

Proof. Denote $A_{F} \triangleq A-\alpha B\left(B^{\mathrm{T}} P B+1\right)^{-1} B^{\mathrm{T}} P A$, and $\beta \triangleq-\left(B^{\mathrm{T}} P B\right) \alpha^{2}+2\left(B^{\mathrm{T}} P B+1\right) \alpha-$ $\left(B^{\mathrm{T}} P B+1\right)$. It can be easily verified that

$$
P-A_{F}^{\mathrm{T}} P A_{F}=Q(\varepsilon)+\frac{\beta}{\left(B^{\mathrm{T}} P B+1\right)^{2}} \cdot A^{\mathrm{T}} P B B^{\mathrm{T}} P A .
$$

Noting that $\beta \geq 0$ for $\alpha \in\left[\alpha_{1}(\varepsilon), \alpha_{2}(\varepsilon)\right]$, one has $P-A_{F}^{\mathrm{T}} P A_{F} \succeq Q(\varepsilon) \succ 0$. Therefore, by the property of Lyapunov equation, $A_{F}$ is Schur. 
The high-low gain design of a bounded control protocol for discrete-time system (1) is performed in three steps.

Algorithm 7: Event-based high-low gain stabilization:

Step 1. Let $\alpha_{1}(\varepsilon)$ be defined in (59). For any initial state $x_{0}$, the low-gain parameter is set as $\varepsilon=\varepsilon_{0}$ :

$$
\varepsilon_{0} \triangleq \max \left\{\varepsilon \in(0,1]: \alpha_{1}(\varepsilon)^{2} \cdot x_{0}^{\mathrm{T}} P x_{0} \cdot\left(1-\frac{\lambda_{1}(Q(\varepsilon))}{\|P\|}\right) \leq \varpi^{2}\right\}
$$

Find $P=P\left(\varepsilon_{0}\right) \succ 0$ to solve ARE (58) with $\varepsilon=\varepsilon_{0}$. Denote

$$
K \triangleq-\left(B^{\mathrm{T}} P B+1\right)^{-1} B^{\mathrm{T}} P A .
$$

Step 2. For $x\left(t_{k}\right) \in \mathcal{T}_{i}$, a high-gain parameter $\alpha(t)$ is defined:

$$
\alpha\left(t_{k}\right) \triangleq \max \left\{\alpha \in\left[\alpha_{1}\left(\varepsilon_{0}\right), \alpha_{2}\left(\varepsilon_{0}\right)\right]:\left|\alpha K x\left(t_{k}\right)\right| \leq \varpi\right\} .
$$

The event-triggering function is designed as follows:

$$
\begin{aligned}
f(t)= & \max \left\{f_{1}(t), f_{2}(t)\right\}, \\
f_{1}(t) \triangleq & -\sigma x(t)^{\mathrm{T}} Q\left(\varepsilon_{0}\right) x(t)-\sigma \beta\left(t_{k}\right) x(t)^{\mathrm{T}} K^{\mathrm{T}} K x(t) \\
& \quad+\alpha\left(t_{k}\right)^{2} e(t)^{\mathrm{T}} K^{\mathrm{T}} B^{\mathrm{T}} P B K e(t)-\gamma\left(t_{k}\right) x(t)^{\mathrm{T}} K^{\mathrm{T}} K e(t), \\
f_{2}(t) \triangleq & \|K e(t)\|-\theta\|K x(t)\|,
\end{aligned}
$$

where the event-trigger parameters $\sigma, \theta \in(0,1)$ are appropriately selected, and $\beta\left(t_{k}\right) \triangleq$ $-\left(B^{\mathrm{T}} P B\right) \alpha\left(t_{k}\right)^{2}+2\left(B^{\mathrm{T}} P B+1\right) \alpha\left(t_{k}\right)-\left(B^{\mathrm{T}} P B+1\right) \geq 0, \gamma\left(t_{k}\right) \triangleq 2 \alpha\left(t_{k}\right)\left(B^{\mathrm{T}} P B\right)\left[\alpha_{2}\left(\varepsilon_{0}\right)-\alpha\left(t_{k}\right)\right]$.

Step 3. A feedback law using $x\left(t_{k}\right)$ is designed as

$$
u(t)=-\alpha\left(t_{k}\right) K x\left(t_{k}\right), t \in\left[t_{k}, t_{k+1}\right), k \geq 0 .
$$

Theorem 4: Consider discrete-time system (1) subject to actuator saturation with saturation threshold $\varpi>0$. Let Assumption 2 hold and $B \in \mathbb{R}^{n}$. Then, Algorithms 5 and 7 can achieve global exponential stabilization of system (1). That is, for any initial state $x(0) \in \mathbb{R}^{n}$, and any $\sigma, \theta \in(0,1)$ in the event-triggering function (63), $\lim _{t \rightarrow \infty} x(t)=0$ is achieved exponentially.

Proof. By (1), (2) and (64), one has $u(t)=-\alpha\left(t_{k}\right) K(e(t)+x(t))$. When saturation does not occur, one has $x(t+1)=A_{F} x(t)+B_{F} e(t)$, where $A_{F} \triangleq A-\alpha\left(t_{k}\right) B K$ and $B_{F} \triangleq-\alpha\left(t_{k}\right) B K$. Consider the Lyapunov function $V(x(t)) \triangleq x^{\mathrm{T}} P x$. Similar to (53), one can evaluate $\Delta V(t)=$ $V(x(t+1))-V(x(t))$ as follows:

$$
\begin{aligned}
\Delta V(t)= & x^{\mathrm{T}}\left(A_{F}^{\mathrm{T}} P A_{F}-P\right) x+e^{\mathrm{T}} B_{F}^{\mathrm{T}} P B_{F} e-\gamma\left(t_{k}\right) x^{\mathrm{T}} K^{\mathrm{T}} K e \\
= & -x^{\mathrm{T}} Q\left(\varepsilon_{0}\right) x-\beta\left(t_{k}\right) x^{\mathrm{T}} K^{\mathrm{T}} K x \\
& +\alpha\left(t_{k}\right)^{2} e^{\mathrm{T}} K^{\mathrm{T}} B^{\mathrm{T}} P B K e-\gamma\left(t_{k}\right) x^{\mathrm{T}} K^{\mathrm{T}} K e \\
= & f_{1}(t)-(1-\sigma) x^{\mathrm{T}} Q\left(\varepsilon_{0}\right) x-(1-\sigma) \beta\left(t_{k}\right)\|K x\|^{2} .
\end{aligned}
$$


The event-trigger strategy in Algorithm 5 enforces $f_{1}(t) \leq 0$. The remaining of the proof is similar to that of Theorem 3.

\section{Numerical Examples}

In this section, numerical examples are presented to verify the theoretical results and to illustrate the effectiveness of the proposed event-based control algorithms. Simulations are performed for: (i) the double integrators in Example 1; (ii) a continuous-time system with a pair of double defective eigenvalues on the imaginary axis in Example 2; and (iii) a linear system model of the inverted pendulum in Example 3.

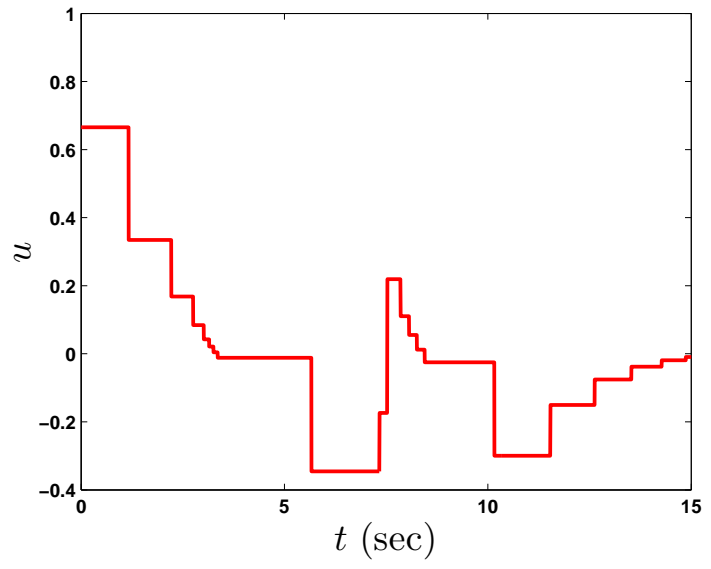

(a) $u$

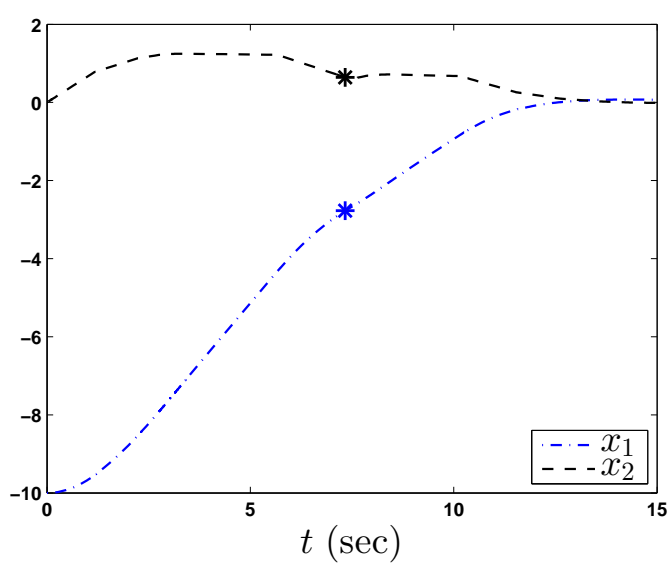

(b) The state trajectories

Figure 1. Event-based high-low gain control for double integrators: (a) the bounded control input; (b) the state trajectories.

Example 1: Consider system (1) as the continuous-time input-saturated double integrators with

$$
A=\left[\begin{array}{ll}
0 & 1 \\
0 & 0
\end{array}\right], \quad B=\left[\begin{array}{l}
0 \\
1
\end{array}\right], \quad \varpi=1
$$

The high-low gain ETC algorithms in Section 3.4 are applied to the initial condition $x(0)=$ $[-10,0]^{\mathrm{T}}$. Following (Grognard et al., 2002, Eq. (18)), the matrix $Q(\varepsilon)$ used for ARE $(24)$ is in the following form:

$$
Q(\varepsilon)=\left[\begin{array}{cc}
\varepsilon^{4} & 0 \\
0 & \varepsilon^{2}
\end{array}\right]
$$

In (24), we let $r=15$. The event-trigger parameters in Algorithm 4 are chosen as $\sigma=\theta=0.99$. The low-gain parameter $\varepsilon$ is selected as $\varepsilon_{0}=0.5076$ following (26). By (31), the controller matrices are obtained with $K_{0}=[0.0665,0.3876]$ and $K_{1}=[0.2582,0.7636]$. When the state $x\left(t_{k}\right)$ is located in the set $\mathcal{T}_{0}$, the high-gain parameter $\alpha\left(t_{k}\right)$ in (29) and the minimum inter-event time $\tau_{k}$ in (38) are on-line scheduled. After $x\left(t_{k}\right)$ reaches the set $\mathcal{T}_{1}$, the minimum inter-event time is set a priori as $\tilde{\tau}=0.19$ sec. For the initial value $x(0)=[-10,0]^{\mathrm{T}}$, the simulation results are shown in Fig. 1. Practical stabilization is achieved within $15 \mathrm{sec}$. The total number of event-based feedback updates is 21. The minimum inter-event time for the 21 updates is $0.10 \mathrm{sec}$. The stars in Fig. 1(b) indicate that it takes $7.33 \mathrm{sec}$ for the state trajectories to reach the set $\mathcal{T}_{1}$ from $x(0)$, to be compared with $6.79 \mathrm{sec}$ for the algorithm in Grognard et al. (2002) using continuous-time feedback control updating. 
Meanwhile, for double integrators subject to actuator saturation, the global stabilization can be achieved via linear event-triggered control law. Applying the global ETC algorithm in Zhang \& Chen (2015), there are 12 event-triggered updates within 15 seconds of simulation. The minimum inter-event time for the 12 updates is $0.7 \mathrm{sec}$. To demonstrate the advantage of the low-gain results in this paper, a system with double defective open-loop poles on the imaginary axis is considered in next example, for which the global ETC algorithm in Zhang \& Chen (2015) is not applicable.

Example 2: Consider system (1) as a continuous-time input-saturated system with saturation threshold $\varpi=1$ and

$$
A=\left[\begin{array}{cccc}
0 & 1 & 1 & 0 \\
-1 & 0 & 0 & 1 \\
0 & 0 & 0 & 1 \\
0 & 0 & -1 & 0
\end{array}\right], \quad B=\left[\begin{array}{l}
0 \\
0 \\
0 \\
1
\end{array}\right]
$$

which has double defective open-loop poles at $\{j, j,-j,-j\}$. The system matrices are equivalent to those in (Lin, 1999, Example 2.2.1) by similarity transformation.

The high-low gain ETC algorithms in Section 3.4 are applied to the initial condition $x(0)=$ $[-10,0,0,0]^{\mathrm{T}}$. The matrix $Q(\varepsilon)$ used for $\operatorname{ARE}(24)$ is in the form of $Q(\varepsilon)=\varepsilon I_{4}$. To illustrate the characteristics of our ETC algorithms, we consider three cases with different parameters as shown in Table 1.

\begin{tabular}{|c|c|c|c|c|c|c|}
\hline Case & $r$ & $\sigma$ & $\theta$ & $\varepsilon_{0}$ & $t_{r}$ & $N$ \\
\hline (i) & 1 & 0.99 & 0.99 & 0.0023 & 24 & 39 \\
\hline (ii) & 15 & 0.99 & 0.99 & 0.0359 & 12.71 & 33 \\
\hline (iii) & 15 & 0.01 & 0.01 & 0.0359 & 12.62 & 354 \\
\hline
\end{tabular}

Table 1. Three cases with different parameters

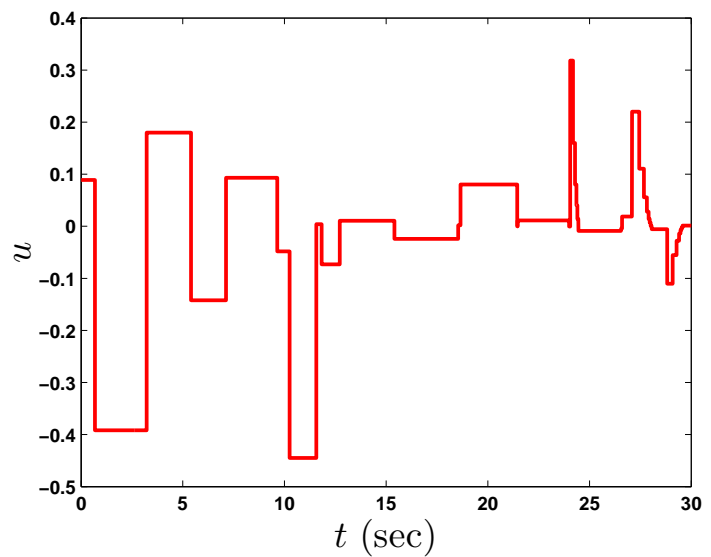

(a) $u$

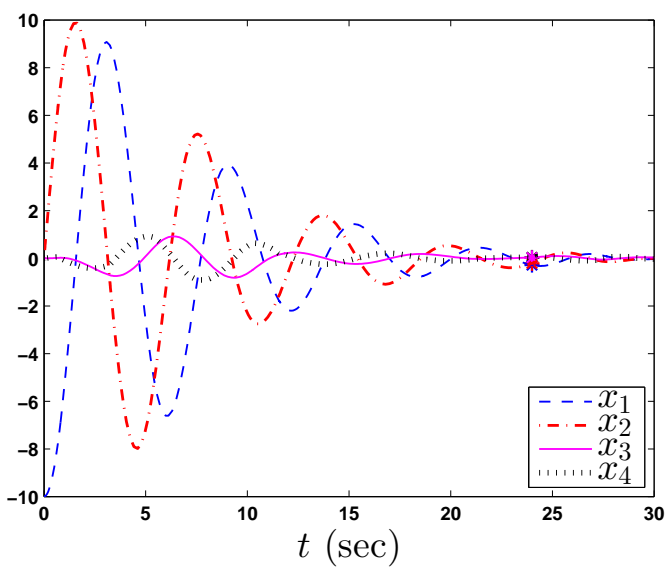

(b) The state trajectories

Figure 2. Event-based high-low gain control for Example 2 Case (i): (a) the bounded control input; (b) the state trajectories.

If $r=1, \sigma=\theta=0.99$, the low-gain parameter $\varepsilon$ is selected as $\varepsilon_{0}=0.0023$ following (26). By (31), one obtains that $K_{0}=[0.0089,0.0672,0.0695,0.5252]$ and $K_{1}=[0.8178,1.1538,1.8858,2.6607]$. When $x\left(t_{k}\right) \in \mathcal{T}_{0}, \alpha\left(t_{k}\right)$ and $\tau_{k}$ are on-line scheduled. After $x\left(t_{k}\right)$ reaches $\mathcal{T}_{1}$, the minimum interevent time is set a priori as $\tilde{\tau}=0.05 \mathrm{sec}$. The simulation results are shown in Fig. 2, where the practical stabilization is achieved within $30 \mathrm{sec}$. It takes $t_{r}=24 \mathrm{sec}$ for the state trajectories to reach $\mathcal{T}_{1}$ from $x(0)$. The total number of event-based updates is $N=39$. The minimum inter-event time for the 39 updates is $0.02 \mathrm{sec}$. 


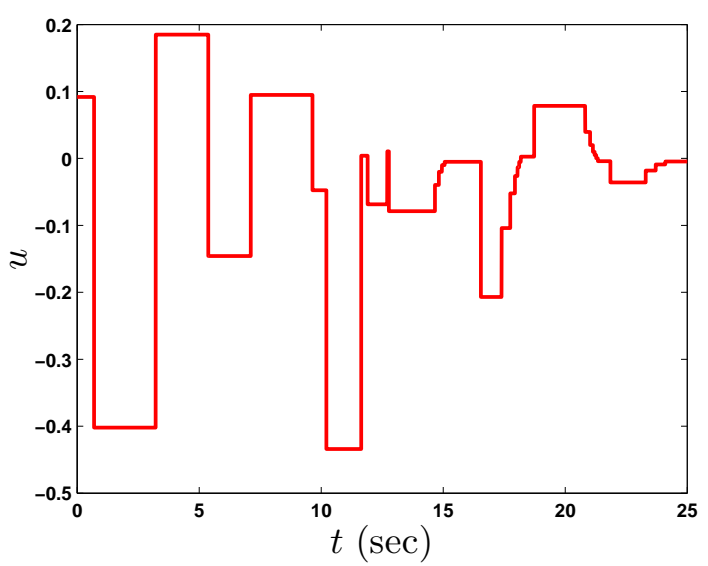

(a) $u$

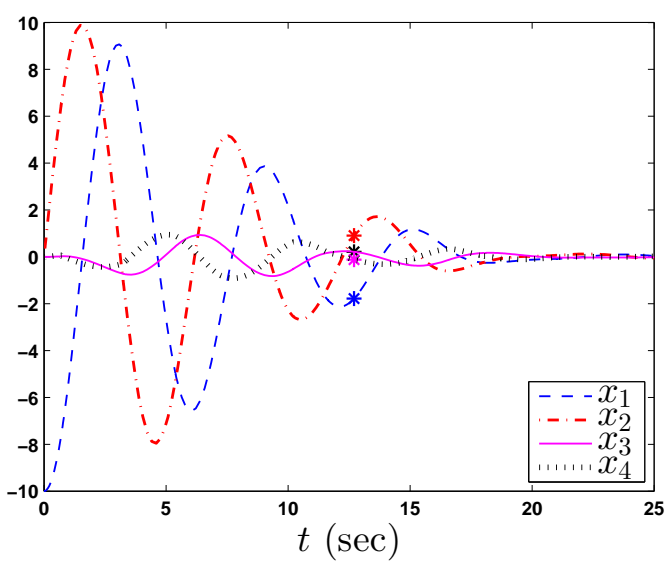

(b) The state trajectories

Figure 3. Event-based high-low gain control for Example 2 Case (ii): (a) the bounded control input; (b) the state trajectories.

If $r=15, \sigma=\theta=0.99$, we let $\varepsilon_{0}=0.0359, K_{0}=[0.0092,0.0686,0.0710,0.5305]$, and $K_{1}=$ $[0.1135,0.3471,0.4116,1.2586]$. When $x\left(t_{k}\right) \in \mathcal{T}_{0}, \alpha\left(t_{k}\right)$ and $\tau_{k}$ are on-line scheduled. After $x\left(t_{k}\right)$ reaches $\mathcal{T}_{1}$, the minimum inter-event time is set a priori as $\tilde{\tau}=0.066 \mathrm{sec}$. The simulation results are shown in Fig. 3, where the practical stabilization is achieved within $25 \mathrm{sec}$. It takes $t_{r}=12.71 \mathrm{sec}$ for the state trajectories to reach $\mathcal{T}_{1}$ from $x(0)$. The total number of event-based updates is $N=33$. The minimum inter-event time for the 33 updates is 0.066 sec.

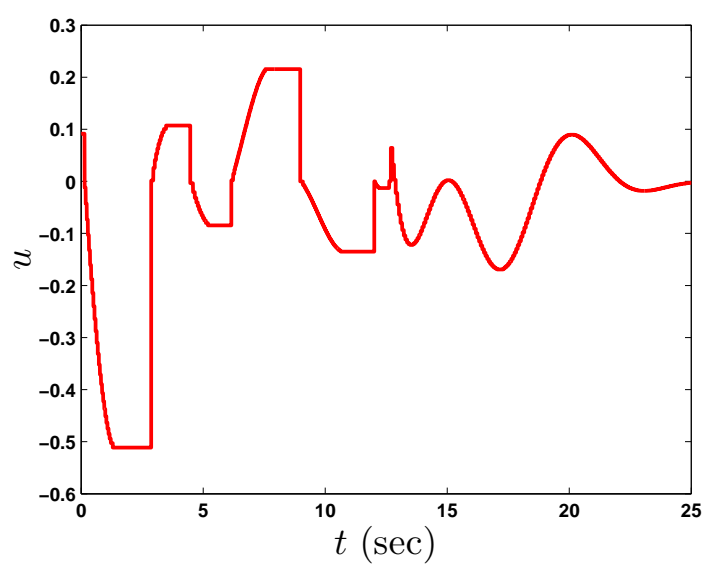

(a) $u$

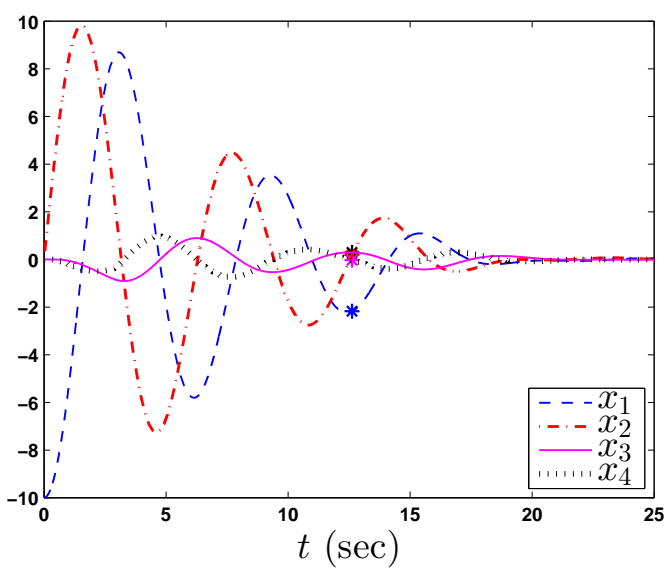

(b) The state trajectories

Figure 4. Event-based high-low gain control for Example 2 Case (iii): (a) the bounded control input; (b) the state trajectories.

If $r=15, \sigma=\theta=0.01$, the simulation results are shown in Fig. 4. It takes $t_{r}=12.62 \mathrm{sec}$ for the state trajectories to reach $\mathcal{T}_{1}$ from $x(0)$. The total number of event-based updates is $N=354$. The minimum inter-event time for the 354 updates is 0.017 sec. In Fig. 4(a), the control input updating is nearly continuous-time after $15 \mathrm{sec}$; while the control performance in Fig. 4(b) is not essentially improved than that in Fig. 3(b).

It is shown that increasing the value of the ARE parameter $r$ can obtain a faster convergence. However, by (44), if $r$ is too large, an extremely small $\tilde{\tau}$ will be obtained and the updating can be very frequent. Furthermore, although the event-trigger parameters can be freely selected without losing stability, small value of $\sigma, \theta$ can lead to frequent control updating.

Example 3: Consider a linear system model of the inverted pendulum as shown in Fig. 5. Denote $x_{1}=\phi$ and $x_{2}=\dot{\phi}$. Following Wu et al. $(2015,2014)$, we let $|u| \leq 1, M=m=0.1 \mathrm{~kg}$, and 


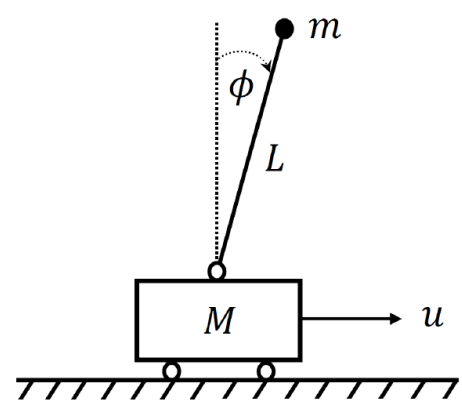

Figure 5. The inverted pendulum.

$L=0.545 \mathrm{~m}$. Compared with the simple event rule $\left\|x-x\left(t_{k}\right)\right\| \leq \sigma\|x\|$, the advantage of the event-triggering condition defined by two functions in (30) and (63) will be investigated. When the angle $\phi$ is small, the dynamics can be described by continuous-time system (1) with

$$
A=\left[\begin{array}{cc}
0 & 1 \\
36 & 0
\end{array}\right], \quad B=\left[\begin{array}{c}
0 \\
-18.3486
\end{array}\right], \quad \varpi=1 .
$$

This is a strictly unstable system with open-loop poles \pm 6 . The initial value $x(0)=[0.02,0.8]^{\mathrm{T}}$ is considered such that the ARE-based algorithms designed for marginally stable systems are still applicable.

Applying the high-low gain ETC algorithms in Section 3.4, we let $Q(\varepsilon)=\varepsilon I_{2}, r=1, \varepsilon_{0}=0.8453$, $\sigma=\theta=0.95, K_{0}=[-4.1287,-1.1381]$ and $K_{1}=[-4.1642,-1.2058]$. The simulation results are shown in Fig. 6(a), where the practical stabilization is achieved within 1.5 sec. The total number of event-based updates is 10 . The minimum inter-event time for the 10 updates is $0.004 \mathrm{sec}$.

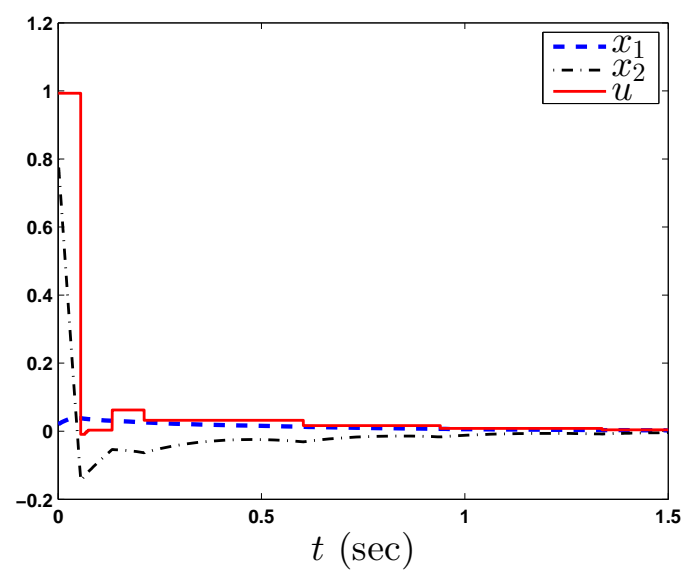

(a) System response by Theorem 2

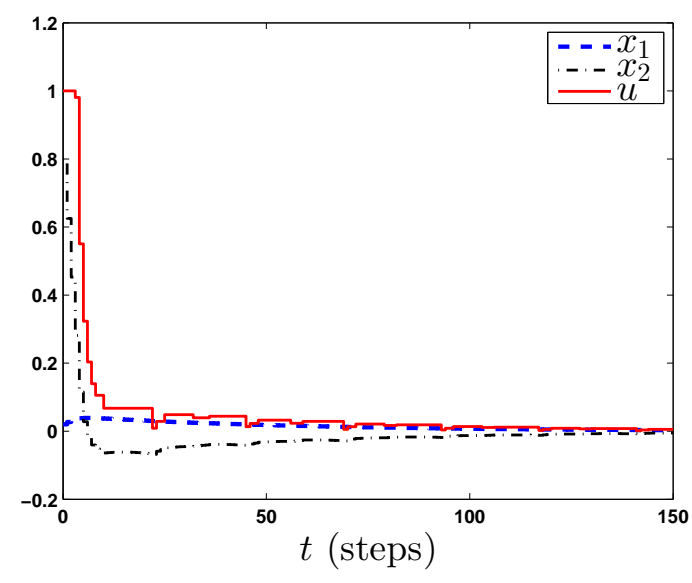

(b) System response by algorithm in Wu et al. (2014)

Figure 6. Event-triggered control for inverted pendulum subject to actuator saturation: (a) system response by Theorem 2 ; (b) system response by algorithm in Wu et al. (2014).

Considering the zero-order-hold discretized system with sampling period 0.01 sec:

$$
A_{d}=\left[\begin{array}{cc}
1.002 & 0.01 \\
0.36 & 1.002
\end{array}\right], \quad B_{d}=\left[\begin{array}{l}
-0.0009 \\
-0.1836
\end{array}\right], \quad \varpi=1,
$$

which is the same as (Wu et al., 2014, Eq. (32)). Applying the algorithm in (Wu et al., 2014, Example 3.2), that is, the event rule $\left\|x-x\left(t_{k}\right)\right\| \leq \sigma\|x\|$ with $\sigma=0.1$ and the control $u(t)=$ $6.3717 x_{1}\left(t_{k}\right)+2.6619 x_{2}\left(t_{k}\right)$, the simulation results are shown in Fig. 6(b), where the practical stabilization is achieved within 150 steps. The total number of event-based updates is 38 . 
Applying the high-low gain ETC algorithms in Section 4.4 to the discretized system, we let $Q(\varepsilon)=\varepsilon I_{2}, \varepsilon_{0}=0.2398, \sigma=\theta=0.99, K=[-3.8529,-0.7828]$. The simulation results are shown in Fig. 7, where the practical stabilization is achieved within 100 steps. The total number of event-based updates is 14 , which is much smaller than the total steps of simulation.

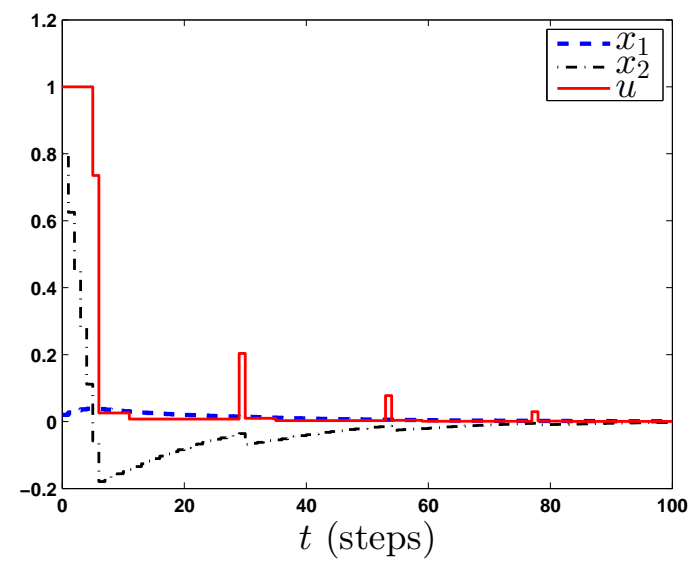

Figure 7. Event-triggered control for inverted pendulum subject to actuator saturation: system response by Theorem 4 .

If there is no saturation constraint, applying the algorithm in (Wu et al., 2014, Example 3.1), that is, the event rule $\left\|x-x\left(t_{k}\right)\right\| \leq \sigma\|x\|$ with $\sigma=0.1$ and the control $u(t)=5.394 x_{1}\left(t_{k}\right)+5.024 x_{2}\left(t_{k}\right)$, the simulation results are shown in Fig. 8, where the fast practical stabilization is achieved within only three steps and the control input is larger than 4 at the beginning. However, with the input saturation constraint $|u| \leq 1$, the practical stabilization cannot be achieved within 50 steps.

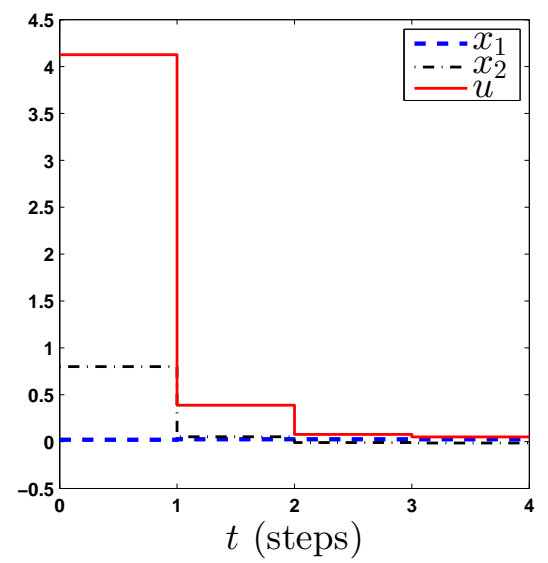

Figure 8. ETC for inverted pendulum subject to actuator saturation: system response with no saturation constraint.

When the system is subject to the actuator saturation constraint, it is shown that the system response by Theorem 2 has the least updates, and the system response by Theorem 4 has the fastest convergence. Compared with the simple event rule $\left\|x-x\left(t_{k}\right)\right\| \leq \sigma\|x\|$, the event-triggering condition defined by two functions in (30) and (63) can further reduce the updating events. In addition, the event-trigger parameters $\sigma, \theta$ in our algorithms can be freely selected while the stability is always theoretically guaranteed by Theorems 2 and 4 .

Remark 6: The research interest in the semi-global ETC is motivated by the ETC studies in (Chen et al., 2015a; Zhang et al., 2014a) and the input saturation studies in (Chen et al., 2015b; Su et al., 2013). When considering the ETC and the input saturation simultaneously, the research difficulties can be noted in both the stability analysis and the numerical examples. From the theoretical point 
of view, it is mathematically involving to perform the Lyapunov stability analysis, see Claims 1-4 in the proof of Theorem 1. It is not straightforward to show that the input saturation is never attained under the low-gain ETC design. From the practical point of view, the issue of actuator saturation can significantly affect the system performance of the ETC design. In Example 3, it is noted that the fast stabilization with no saturation constraint (see Fig. 8) can be lost if there is the input saturation constraint (see Fig. 6(b)). In the future, the semi-global ETC design will be extended to the Markov jump systems (Chen et al., 2014a; Li et al., 2012) and the multi-agent systems (Chen et al., 2014b; Su et al., 2013), where the considered systems will be more complicated.

\section{Conclusion}

In this paper, the problem of event-based stabilization has been investigated for null controllable systems subject to input saturation. For both continuous-time and discrete-time systems, novel event-triggered low-gain control algorithms based on Riccati equations have been proposed to achieve semi-global stabilization. Furthermore, the semi-global results are extended to eventtriggered global stabilization by utilizing the high-low gain techniques. Future studies include the robust event-triggered control for systems with disturbance, the event-triggered control for the output-feedback systems, the optimal event-triggered control considering the control performance, and the extensions of the results to Markov jump systems and multi-agent systems.

\section{Acknowledgment}

The authors would like to thank the Senior Editor and the anonymous reviewers for their insightful suggestions. This work was supported in part by the National Natural Science Foundation of China under Grant 61374053 and in part by the Hong Kong University Committee on Research and Conference Grants under Grant 201309176141.

\section{References}

Ames, A. D., Tabuada, P., \& Sastry, S. (2006). On the stability of Zeno equilibria. In Hybrid Systems: Computation and Control. Lecture Notes in Computer Science, vol. 3927, pp. 34-48. Springer.

Anta, A., \& Tabuada, P. (2010). To sample or not to sample: Self-triggered control for nonlinear systems. IEEE Transactions on Automatic Control, 55 (9), 2030-2042.

Aström, K. J., \& Bernhardsson, B. (1999). Comparison of periodic and event based sampling for first order stochastic systems. In Proceedings of the 14th IFAC World Congress, pp. 301-306, 1999.

Arzén, K. E. (1999). A simple event-based PID controller. In Proceedings of the 14th IFAC World congress, pp. $423-428,1999$.

Bernstein, D., \& Michel, A. (1995). Special issue on saturating actuators. International Journal of Robust and Nonlinear Control, 5(5), 375-540.

Chen, F., Xu, S., Zou, Y., \& Xu, H. (2014a). Controller design for nonlinear quadratic Markov jumping systems with input saturation. International Journal of Control, 87(1), 32-40.

Chen, X., Hao, F., \& Rahmani, A. (2014b). Asynchronous decentralised event-triggered control of multiagent systems. International Journal of Control, 87(10), 2130-2139.

Chen, M. Z. Q., Zhang, L., Su, H., \& Li, C. (2015a). Event-based synchronization of linear discrete-time dynamical networks. IET Control Theory and Applications, vol. 9, no. 5, pp. 755-765, 2015.

Chen, M. Z. Q., Zhang, L., Su, H., \& Chen, G. (2015b). Stabilizing solution and parameter dependence of modified algebraic Riccati equation with application to discrete-time network synchronization. IEEE Transactions on Automatic Control, in press, DOI: 10.1109/TAC.2015.2434011.

Eqtami, A., Dimarogonas, D. V., \& Kyriakopoulos, K. J. (2010). Event-triggered control for discrete-time systems. In Proceedings of the 2010 American Control Conference, pp. 4719-4724, 2010. 
Grognard, F., Sepulchre, R., \& Bastin, G. (2002). Improving the performance of low-gain designs for bounded control of linear systems. Automatica, 38(10), 1777-1782.

Gupta, S. (1963). Increasing the sampling efficiency for a control system. IEEE Transactions on Automatic Control, 8(3), 263-264.

Heemels, W. P. M. H., Sandee, J. H., \& van den Bosch, P. P. J. (2008). Analysis of event-driven controllers for linear systems. International Journal of Control, 81 (4), 571-590.

Heemels, W. P. M. H., Donkers, M. C. F., \& Teel, A. R. (2013). Periodic event-triggered control for linear systems. IEEE Transactions on Automatic Control, 58(4), 847-861.

Hille, E. (1969). Lectures on Ordinary Differential Equations. Addison-Wesley.

Kiener, G. A., Lehmann, D., \& Johansson, K. H. (2014). Actuator saturation and anti-windup compensation in event-triggered control. Discrete Event Dynamic Systems, 24(2), 173-197.

Lehmann, D., \& Johansson, K. H. (2012). Event-triggered PI control subject to actuator saturation. In Proceedings of the 2nd IFAC Conference on Advances in PID Control, pp. 430-435, Brescia, Italy, 2012.

Li, C., Chen, M. Z. Q., Lam, J., \& Mao, X. (2012). On exponential almost sure stability of random jump systems. IEEE Transactions on Automatic Control, 57(12), 3064-3077.

Lin, Z., Saberi, A., \& Stoorvogel, A. A. (1996). Semiglobal stabilization of linear discrete-time systems subject to input saturation via linear feedback-An ARE-based approach. IEEE Transactions on Automatic Control, $41(8), 1203-1207$.

Lin, Z. (1999). Low Gain Feedback. London: Springer-Verlag.

Mazo, M. Jr., \& Tabuada, P. (2011). Decentralized event-triggered control over wireless sensor/actuator networks. IEEE Transactions on Automatic Control, 56(10), 2456-2461.

Michel, A. N., Hou, L., \& Liu, D. (2008). Stability of Dynamical Systems: Continuous, Discontinuous, and Discrete Systems. Boston: Birkhäuser.

Ni, W., Zhao, P., Wang, X., \& Wang, J. (2015). Event-triggered control of linear systems with saturated inputs. Asian Journal of Control, 17(4), 1196-1208.

Shi, P., Yin, Y., \& Liu, F. (2013). Gain-scheduled worst-case control on nonlinear stochastic systems subject to actuator saturation and unknown information. Journal of Optimization Theory and Applications, $156(3), 156(3), 844-858$.

Su, H., Chen, M. Z. Q., Lam, J., \& Lin, Z. (2013). Semi-global leader-following consensus of linear multiagent systems with input saturation via low gain feedback. IEEE Transactions on Circuits and Systems I: Regular Papers, 60(7), 1881-1889.

Su, H., Chen, M. Z. Q., Wang, X., \& Lam, J. (2014). Semi-global observer-based leader-following consensus with input saturation. IEEE Transactions on Industrial Electronics, 61 (6), 2842-2850.

Tabuada, P. (2007). Event-triggered real-time scheduling of stabilizing control tasks. IEEE Transactions on Automatic Control, 52(9), 1680-1685.

Teel, A. R. (1992). Global stabilization and restricted tracking for multiple integrators with bounded controls. Systems \& Control Letters, 18, 165-171.

Teel, A. R. (1995). Semi-global stabilizability of linear null controllable systems with input nonlinearities. IEEE Transactions on Automatic Control, 40(1), 96-100.

Wang, X., \& Lemmon, M. D. (2009). Self-triggered feedback control systems with finite-gain stability. IEEE Transactions on Automatic Control, 54 (3), 452-467.

Wang, X., \& Lemmon, M. D. (2011). Event-triggering in distributed networked control systems. IEEE Transactions on Automatic Control, 56(3), 586-601.

Wang, C., Yu, X., \& Lan, W. (2014). Semi-global output regulation for linear systems with input saturation by composite nonlinear feedback control. International Journal of Control, 87 (10), 1985-1997.

Wu, W., Reimann, S., Gorges, D., \& Liu, S. (2015). Event-triggered control for discrete-time linear systems subject to bounded disturbance. International Journal of Robust and Nonlinear Control, in press, DOI: 10.1002/rnc.3388.

Wu, W., Reimann, S., \& Liu, S. (2014). Event-triggered control for linear systems subject to actuator saturation. In Proceedings of the 19th IFAC World Congress, pp. 9492-9497, Cape Town, South Africa, 2014.

Yang, Y., Sontag, E. D., \& Sussmann, H. J. (1997). Global stabilization of linear discrete-time systems with bounded feedback. Systems \& Control Letters, 30 (5), 273-281.

Zhang, L. (2015). Riccati equation and event-triggered control for constrained systems. PhD thesis, The University of Hong Kong, Hong Kong, 2015.

Zhang, L., \& Chen, M. Z. Q. (2015). Event-based global stabilization of linear systems via a saturated linear 
controller. International Journal of Robust and Nonlinear Control, in press, DOI: 10.1002/rnc.3352.

Zhang, L., Chen, M. Z. Q., Li, C., \& Shu, Z. (2014a). Event-triggered control over noisy feedback channels. In Proceedings of the 19th IFAC World Congress, pp. 10493-10498, Cape Town, South Africa, 2014.

Zhang, Z., Hao, F., Zhang, L., \& Wang, L. (2014b). Consensus of linear multi-agent systems via eventtriggered control. International Journal of Control, 87(6), 1243-1251.

Zuo, Z., Wang, Y., \& Yang, W. (2015). $L_{2}$-gain fault tolerant control of singular Lipschitz systems in the presence of actuator saturation. International Journal of Robust and Nonlinear Control, 25(12), 17511766.

\section{Appendix A. Two Lemmas used for Proof of Theorem 1}

Lemma 8: Let $\phi(t), t \geq \tilde{t}$, be a real-valued function, and $a_{2}, a_{1}, a_{0}$ be positive constants satisfying $a_{1}^{2}-4 a_{2} a_{0} \geq 0$. Denote $\mu \triangleq \sqrt{a_{1}^{2}-4 a_{2} a_{0}}$. Consider the Riccati equation (Hille, 1969)

$$
\frac{d}{d t} \phi=a_{2} \phi^{2}+a_{1} \phi+a_{0}
$$

with zero initial condition $\phi(\tilde{t})=0$. It has a unique solution as

$$
\phi(t)=\frac{b_{1}\left(1-e^{-\mu(t-\tilde{t})}\right)}{e^{-\mu(t-\tilde{t})}-b_{2}}, \quad t \in\left[\tilde{t}, \tilde{t}+\frac{1}{\mu} \ln \frac{1}{b_{2}}\right)
$$

if $\mu>0$, where $b_{1} \triangleq \frac{a_{1}-\mu}{2 a_{2}}$ and $b_{2} \triangleq \frac{a_{1}-\mu}{a_{1}+\mu}$; for $\forall \bar{\phi}>0$, there exists a unique $\bar{\tau} \in\left[0, \frac{1}{\mu} \ln \frac{1}{b_{2}}\right)$ such that $\phi(\tilde{t}+\bar{\tau})=\bar{\phi}$. If $\mu=0$, the unique solution is

$$
\phi(t)=\frac{1}{a_{2}\left(\frac{2}{a_{1}}+\tilde{t}-t\right)}-\frac{a_{1}}{2 a_{2}}, \quad t \in\left[\tilde{t}, \frac{2}{a_{1}}+\tilde{t}\right)
$$

and there exists a unique $\bar{\tau} \in\left[0, \frac{2}{a_{1}}\right)$ such that $\phi(\tilde{t}+\bar{\tau})=\bar{\phi}$ for any $\bar{\phi}>0$.

Proof. Denote $\psi=\phi+\frac{a_{1}}{2 a_{2}}>\frac{\mu}{2 a_{2}}$ and $\psi(\tilde{t})=\frac{a_{1}}{2 a_{2}}$. One has

$$
\frac{d \psi}{d t}=a_{2} \psi^{2}-\frac{\mu^{2}}{4 a_{2}}=a_{2}\left(\psi+\frac{\mu}{2 a_{2}}\right)\left(\psi-\frac{\mu}{2 a_{2}}\right) .
$$

If $\mu>0$, then

$$
\begin{aligned}
& \int \frac{\mu^{2}}{a_{2}} d t=\int \frac{1}{\left(\frac{a_{2}}{\mu} \psi+\frac{1}{2}\right)\left(\frac{a_{2}}{\mu} \psi-\frac{1}{2}\right)} d \psi ; \\
& \frac{\mu^{2}}{a_{2}}(t-\tilde{t})=\frac{\mu}{a_{2}} \ln \frac{\left(\psi-\frac{\mu}{2 a_{2}}\right)\left(a_{1}+\mu\right)}{\left(\psi+\frac{\mu}{2 a_{2}}\right)\left(a_{1}-\mu\right)} \\
& \psi=\frac{\mu}{2 a_{2}} \cdot \frac{e^{-\mu(t-\tilde{t})}+b_{2}}{e^{-\mu(t-\tilde{t})}-b_{2}}
\end{aligned}
$$

Thus, $\phi(t)$ is solved as in (A1). Since $\phi \geq 0, e^{-\mu(t-\tilde{t})}-b_{2}>0$, that is,

$$
0 \leq t-\tilde{t}<\frac{1}{\mu} \ln \frac{1}{b_{2}} .
$$


In fact, one has

$$
\lim _{t \rightarrow\left(\tilde{t}+\frac{1}{\mu} \ln \frac{1}{b_{2}}\right)+0^{-}} \phi(t)=+\infty
$$

Then, for $\forall \bar{\phi}>0$, since $\phi(\tilde{t})=0$ and $\frac{d}{d t} \phi(t)>0$, it is straightforward to verify that there exists a unique $\bar{\tau}<\frac{1}{\mu} \ln \frac{1}{b_{2}}$ such that $\phi(\tilde{t}+\bar{\tau})=\bar{\phi}$.

If $\mu=0$, one has

$$
\int \frac{1}{\psi^{2}} d \psi=\int a_{2} d t ; \quad \frac{2 a_{2}}{a_{1}}-\frac{1}{\psi}=a_{2}(t-\tilde{t}) ; \quad \psi=\frac{1}{a_{2}\left(\frac{2}{a_{1}}+\tilde{t}-t\right)}
$$

and for $\forall \bar{\phi}>0$, the unique

$$
\bar{\tau}=\frac{2}{a_{1}}-\frac{2}{\left(a_{1}+2 a_{2} \bar{\phi}\right)}
$$

makes $\phi(\tilde{t}+\bar{\tau})=\bar{\phi}$

Lemma 9: Let $y(t):(\tilde{t},+\infty) \rightarrow \mathbb{R}^{q}$ be a continuously differentiable map and has the right derivative $y_{+}^{\prime}(\tilde{t})=\lim _{t \rightarrow \tilde{t}^{+}} \dot{y}(t)$; denote $\varphi(t) \triangleq\|y(t)\|$. Then, for $t>\tilde{t}$, one has that

- (i) $\dot{\varphi}(t)=\frac{1}{\|y\|} y^{\mathrm{T}} \dot{y}$ if $y(t) \neq 0$;

- (ii) $D \varphi(t) \leq \sqrt{2}\|\dot{y}\|$ if $y(t)=0$, where $D \varphi(t)$ denotes the Dini derivative of $\varphi(t)$;

- (iii) if $y(t)=0$ and the second-order derivative $\ddot{y}(t)$ exists, then $D \varphi(t) \leq\|\dot{y}(t)\|$.

Furthermore, one has

- (iv) $\varphi_{+}^{\prime}(\tilde{t})=\frac{1}{\|y(\tilde{t})\|} y(\tilde{t})^{\mathrm{T}} y_{+}^{\prime}(\tilde{t})$ if $y(\tilde{t}) \neq 0$;

- $(\mathrm{v}) D^{+} \varphi(\tilde{t}) \leq \sqrt{2}\left\|y_{+}^{\prime}(\tilde{t})\right\|$ if $y(\tilde{t})=0$;

- (vi) if $y(\tilde{t})=0$ and the second-order right derivative $y_{+}^{\prime \prime}(\tilde{t})$ exists, then $D^{+} \varphi(\tilde{t}) \leq\left\|y_{+}^{\prime}(\tilde{t})\right\|$.

Proof. (i) If $y(t) \neq 0$, one has

$$
\frac{d}{d t}\|y(t)\|=\frac{d}{d t} \sqrt{y^{\mathrm{T}} y}=\frac{1}{2 \sqrt{y^{\mathrm{T}} y}} \cdot \frac{d}{d t}\left(y^{\mathrm{T}} y\right)=\frac{1}{\|y\|} y^{\mathrm{T}} \dot{y} .
$$

(ii) If $y(t)=0$, denote $\zeta(h) \triangleq y(t+h)^{\mathrm{T}} y(t+h) ; \zeta(0)=0, \zeta^{\prime}(0)=0$. Applying the Taylor series with the Lagrange remainder, one has

$$
\zeta(h)=\zeta^{\prime}\left(\mu_{1} h\right) h=2 y\left(t+\mu_{1} h\right)^{\mathrm{T}} \dot{y}\left(t+\mu_{1} h\right) h,
$$

where $\mu_{1} \in[0,1]$; and $y\left(t+\mu_{1} h\right)=\dot{y}\left(t+\mu_{2} \mu_{1} h\right) \mu_{1} h$, where $\mu_{2} \in[0,1]$. Then,

$$
\zeta(h)=2 \mu_{1} h^{2} \dot{y}\left(t+\mu_{2} \mu_{1} h\right)^{\mathrm{T}} \dot{y}\left(t+\mu_{1} h\right) .
$$

Since $0 \leq \mu_{1} \leq 1$, one has

$$
D_{+} \varphi(t) \leq D^{+} \varphi(t)=\varlimsup_{h \rightarrow 0^{+}} \sqrt{\zeta(h)} / h \leq \sqrt{2}\|\dot{y}(t)\| ; D_{-} \varphi(t) \leq D^{-} \varphi(t) \leq 0 .
$$

(iii) If $\ddot{y}$ exists, then one has $\zeta(h)=\frac{1}{2} h^{2} \zeta^{\prime \prime}\left(\mu_{3} h\right)=h^{2} y\left(t+\mu_{3} h\right)^{\mathrm{T}} \ddot{y}\left(t+\mu_{3} h\right)+h^{2}\left\|\dot{y}\left(t+\mu_{3} h\right)\right\|^{2}$, $\lim _{h \rightarrow 0} \zeta(h) / h^{2}=\|\dot{y}(t)\|^{2}$, and $D \varphi(t) \leq\|\dot{y}(t)\|$.

One can obtain (iv)-(vi) similarly to (i)-(iii). 


\section{Appendix B. Proof of Claims 3 and 4}

\section{B.1 For Claim 3}

If no saturation occurs and $x \neq 0$, then $x$ and $e$ are twice differentiable on $\left(t_{k}, t_{k+1}\right)$, have secondorder right derivatives $x_{+}^{\prime \prime}$ and $e_{+}^{\prime \prime}$ at $t_{k}$, and are continuous at $t_{k+1}$. Applying Lemma 9, one obtains that $\|x\|$ is differentiable on $\left(t_{k}, t_{k+1}\right)$ and has right derivative at $t_{k} ; x_{+}^{\prime}\left(t_{k}\right)=A_{F} x\left(t_{k}\right)$, and

$$
\left\|x\left(t_{k}\right)\right\|_{+}^{\prime}=\frac{1}{\left\|x\left(t_{k}\right)\right\|} x^{\mathrm{T}}\left(t_{k}\right) x_{+}^{\prime}\left(t_{k}\right)
$$

By (2), one has $e_{+}^{\prime}\left(t_{k}\right)=-A_{F} x\left(t_{k}\right)$ and $D^{+}\left\|e\left(t_{k}\right)\right\| \leq\left\|A_{F} x\left(t_{k}\right)\right\|$.

Define a real-valued function $z(t)$ of time $t$ as

$$
z(t)=\frac{\|e(t)\|}{\|x(t)\|}, t \in\left[t_{k}, t_{k+1}\right) ; \quad z\left(t_{k}\right)=0 .
$$

From the above analysis, one has

$$
D^{+} z\left(t_{k}\right) \leq \frac{\left\|A_{F} x\left(t_{k}\right)\right\|}{\left\|x\left(t_{k}\right)\right\|} \leq\left\|A_{F}\right\|=a_{0} .
$$

When $t \in\left(t_{k}, t_{k+1}\right)$, by (7) and Lemma 9, and similar to (Tabuada, 2007, Eq. (11) and (15)), one has

$$
\dot{z}(t) \leq a_{2} z^{2}+a_{1} z+a_{0} \text { if } e(t) \neq 0
$$

and

$$
D z(t) \leq \frac{\|\dot{e}\|}{\|x\|} \leq\left\|A_{F}\right\|=a_{0} \text { if } e(t)=0
$$

As a result, $D^{+} z(t) \leq a_{2} z^{2}+a_{1} z+a_{0}, t \in\left[t_{k}, t_{k+1}\right)$. Applying the comparison principle (Michel et al., 2008, Theorem 3.8.1), and by Lemma 8, one has

$$
z(t) \leq \phi(t) \leq \phi\left(t_{k}+\tau\right)=\delta, \quad t \in\left[t_{k}, t_{k}+\tau\right) .
$$

\section{B.2 For Claim 4}

Since $e\left(t_{k}\right)=0, V\left(x\left(t_{k}\right)\right) \leq \beta \varpi^{2}$, using Claim 2 and similar to (9), one has $\left\|u\left(t_{k}\right)\right\|_{\infty} \leq \varpi$ and $V_{+}^{\prime}<0$ at $t=t_{k}$. Denote

$$
\hat{\tau}_{1} \triangleq \sup \left\{s-t_{k} \mid(16) \text { holds on }\left[t_{k}, s\right], s \leq t_{k}+\tau\right\}
$$

and

$$
\hat{\tau}_{2} \triangleq \sup \left\{s-t_{k} \mid V(x(t)) \leq \beta \varpi^{2} \text { on }\left[t_{k}, s\right], s \leq t_{k}+\tau\right\} .
$$

It is straightforward that $\hat{\tau}_{2}>0$, and $\hat{\tau}_{1}>0$ due to $x\left(t_{k}\right) \neq 0$. Applying Claim 2, one obtains that for all $t \in\left(t_{k}, t_{k}+\min \left\{\hat{\tau}_{1}, \hat{\tau}_{2}\right\}\right)$,

$$
\|\tilde{u}(t)\|_{\infty} \leq \varpi \text {, and } \dot{V} \leq 0 \text { due to (19). }
$$

Furthermore, $\dot{V}<0$ holds on $\left(t_{k}, t_{k}+\hat{\tau}_{0}\right)$ for a sufficiently small $\hat{\tau}_{0}>0$. Thus, at $t=t_{k}+\min \left\{\hat{\tau}_{1}, \hat{\tau}_{2}\right\}$, 


$$
V<V\left(x\left(t_{k}\right)\right) \leq \beta \varpi^{2}
$$

which implies $\hat{\tau}_{1} \leq \hat{\tau}_{2}$.

One has

$$
\text { either } \hat{\tau}_{1}=\tau \text { or } \hat{\tau}_{1}<\tau \text {. }
$$

By (2), the condition $x\left(t_{k}\right) \neq 0$ implies that for $\forall t \in\left[t_{k}, t_{k}+\tau\right)$, either $x(t) \neq 0$ or $e(t) \neq 0$.

Suppose that $x(\bar{t})=0$ for some $\bar{t} \in\left(t_{k}, t_{k}+\tau\right]$. Denote

$$
\hat{\tau}_{3} \triangleq \inf \left\{t-t_{k} \mid x(t)=0, t \in\left(t_{k}, \bar{t}\right]\right\} \text { and } \hat{\tau}_{4} \triangleq \min \left\{\hat{\tau}_{1}, \hat{\tau}_{3}\right\} .
$$

Since $e(t)$ and $x(t)$ are differentiable on $\left(t_{k}, t_{k}+\tau\right)$ and have right derivative at $t=t_{k}$, one has

$$
\hat{\tau}_{3}>0, \hat{\tau}_{4}>0 \text {, and } x(t) \neq 0 \text { for } t \in\left[t_{k}, t_{k}+\hat{\tau}_{3}\right) .
$$

For all $t \in\left[t_{k}, t_{k}+\hat{\tau}_{4}\right), x(t) \neq 0$ and (16) holds. If $\hat{\tau}_{4}=\hat{\tau}_{3}<\hat{\tau}_{1}$, then

$$
x\left(t_{k}+\hat{\tau}_{4}\right)=0 \text { and }\left\|e\left(t_{k}+\hat{\tau}_{4}\right)\right\| \leq 0, e\left(t_{k}+\hat{\tau}_{4}\right)=0,
$$

which is impossible. Therefore, $\hat{\tau}_{3} \geq \hat{\tau}_{4}=\hat{\tau}_{1}$. Similar to Claim 3, by Lemma 8, one obtains

$$
\left\|e\left(t_{k}+\hat{\tau}_{1}\right)\right\| \leq \phi\left(t_{k}+\hat{\tau}_{1}\right)\left\|x\left(t_{k}+\hat{\tau}_{1}\right)\right\| .
$$

Thus, $\hat{\tau}_{1} \geq \tau$ and $\hat{\tau}_{1}=\tau$; otherwise,

$$
\phi\left(t_{k}+\hat{\tau}_{1}\right)<\phi\left(t_{k}+\tau\right)=\delta,
$$

and a contradiction results from the definition of $\hat{\tau}_{1}$. Therefore,

$$
\hat{\tau}_{3}=\tau=\hat{\tau}_{2}=\hat{\tau}_{1},
$$

by which one has $x\left(t_{k}+\tau\right)=0$ and $\lim _{t \rightarrow t_{k}+\tau}\|x\|+\|e\|=0$, which is impossible due to (2). Thus, it is concluded that

$$
x(t) \neq 0, \forall t \in\left[t_{k}, t_{k}+\tau\right] .
$$

Suppose that $\hat{\tau}_{1}<\tau$, which implies

$$
\left\|e\left(t_{k}+\hat{\tau}_{1}\right)\right\|=\delta\left\|x\left(t_{k}+\hat{\tau}_{1}\right)\right\| .
$$

On $\left[t_{k}, t_{k}+\hat{\tau}_{1}\right]$, no saturation occurs and $\|x\|$ is positive. Then, similar to Claim 3 , by Lemma 8 , one obtains

$$
\left\|e\left(t_{k}+\hat{\tau}_{1}\right)\right\| \leq \phi\left(t_{k}+\hat{\tau}_{1}\right)\left\|x\left(t_{k}+\hat{\tau}_{1}\right)\right\|<\phi\left(t_{k}+\tau\right)\left\|x\left(t_{k}+\hat{\tau}_{1}\right)\right\|
$$

and $\delta<\phi\left(t_{k}+\tau\right)$, which is a contradiction. Thus, $\hat{\tau}_{1}=\hat{\tau}_{2}=\tau$, for $\forall t \in\left[t_{k}, t_{k}+\tau\right]$, (16) holds, $V(x(t)) \leq \beta \varpi^{2}$, and $\|\tilde{u}(t)\|_{\infty} \leq \varpi$ due to Claim 2. 